ARTICLE

\title{
Critical role of sphingosine-1-phosphate receptor-2 in the disruption of cerebrovascular integrity in experimental stroke
}

\author{
Gab Seok Kim¹,2, Li Yang 1,2, Guoqi Zhang ${ }^{1,2}$, Honggang Zhao ${ }^{1,2}$, Magdy Selim³, Louise D. McCullough", \\ Michael J. Kluk ${ }^{5} \&$ Teresa Sanchez ${ }^{1,2, \dagger}$
}

The use and effectiveness of current stroke reperfusion therapies are limited by the complications of reperfusion injury, which include increased cerebrovascular permeability and haemorrhagic transformation. Sphingosine-1-phosphate (S1P) is emerging as a potent modulator of vascular integrity via its receptors (S1PR). By using genetic approaches and a S1PR2 antagonist (JTE013), here we show that S1PR2 plays a critical role in the induction of cerebrovascular permeability, development of intracerebral haemorrhage and neurovascular injury in experimental stroke. In addition, inhibition of S1PR2 results in decreased matrix metalloproteinase (MMP)-9 activity in vivo and lower gelatinase activity in cerebral microvessels. S1PR2 immunopositivity is detected only in the ischemic microvessels of wild-type mice and in the cerebrovascular endothelium of human brain autopsy samples. In vitro, S1PR2 potently regulates the responses of the brain endothelium to ischaemic and inflammatory injury. Therapeutic targeting of this novel pathway could have important translational relevance to stroke patients.

\footnotetext{
${ }^{1}$ Department of Emergency Medicine, the Center for Vascular Biology Research, Beth Israel Deaconess Medical Center, Harvard Medical School, Boston, Massachusetts 02215, USA. ${ }^{2}$ Department of Surgery, the Center for Vascular Biology Research, Beth Israel Deaconess Medical Center, Harvard Medical School, Boston, Massachusetts 02215, USA. ${ }^{3}$ Department of Neurology, Beth Israel Deaconess Medical Center, Harvard Medical School, 330 Brookline Avenue, RN-227A, Boston, Massachusetts 02215, USA. ${ }^{4}$ Department of Neurology, University of Connecticut School of Medicine, Farmington, Connecticut 06030, USA. ${ }^{5}$ Department of Pathology, Brigham and Women's Hospital, Harvard Medical School, Boston, Massachusetts 02215, USA. $\dagger$ Present address: Department of Pathology and Laboratory Medicine, Center for Vascular Biology, Weill Cornell Medical College, New York, New York 10065, USA. Correspondence and requests for materials should be addressed to T.S. (email: tes2015@med.cornell.edu).
} 
S troke is a leading cause of death and long-term disability worldwide ${ }^{1}$. Despite many decades of intensive research, therapeutic options for patients presenting with ischaemic stroke are very limited. Neuroprotective agents have been designed to block one or more steps of the neuronal ischaemic cascade. However, none of these drugs unequivocally has shown an improvement in clinical outcomes ${ }^{2,3}$. Currently, stroke therapies aim at establishing early reperfusion by thrombolytic (for example, tissue plasminogen activator) and/ or mechanical recanalization of obstructed blood vessel $/ \mathrm{s}^{4,5}$. However, restoration of blood flow exacerbates brain injury due to increased reactive oxygen species generation and further activation of matrix metalloproteinases (MMP) with subsequent development of vasogenic oedema (increased cerebrovascular permeability) and haemorrhagic transformation ${ }^{5,6}$, two lifethreatening complications, which limit the use and effectiveness of stroke reperfusion strategies. Administration of vasoprotective agents at the time of reperfusion could potentially decrease the risk and the extent of these complications by improving the integrity of the injured cerebral blood vessels, which could ultimately result in improved stroke outcomes.

Ischaemia-reperfusion (I/R) injury induces metabolic stress and inflammatory responses in the neurovascular unit. Although neurons are extremely sensitive to the lack of oxygen and nutrients, I/R injury also compromises the barrier properties of brain endothelial cells, which contributes to neurovascular pathophysiology by allowing the entrance of toxic plasma proteins and blood cells into the brain parenchyma ${ }^{7}$, increasing intracranial pressure and compromising the delivery of nutrients and oxygen. In the acute phase of stroke (minutes to hours), reactive oxygen species and pro-inflammatory cytokines released in the brain parenchyma, such as tumour necrosis factor- $\alpha$ $(\mathrm{TNF}-\alpha)$ or interleukin-1 $\beta$, are known to induce vascular permeability, adhesion molecule expression, and the expression and activity of MMP, which exacerbate blood-brain barrier disruption, worsening neurovascular injury ${ }^{8,9}$. In the subacute phase, further disruption of blood vessel integrity occurs, leading to haemorrhagic transformation and exacerbation of neuronal injury ${ }^{5}$. In experimental models of stroke, activation of MMP and the subsequent degradation of endothelial basal lamina and tight junction proteins have been shown to play a critical role in the disruption of cerebrovascular integrity $8,10,11$. Since MMPs can be highly toxic molecules, they are tightly regulated at the transcriptional, translational and post-translational levels. Among MMP, MMP-2 is constitutively expressed in the brain and brain endothelial cells, while basal MMP-9 levels are very low and rapidly induced by pro-inflammatory stimuli ${ }^{12}$. Although it is well-established that MMP contribute to neurovascular injury in stroke, currently, there are no therapies specifically targeting MMP, due to the complex regulation of MMP activity, as well as the lack of specificity of MMP inhibitors and their numerous side effects.

The bioactive sphingolipid, sphingosine-1-phosphate (S1P) is emerging as a potent modulator of vascular integrity. S1P is present at high levels (within the range of several hundred nanomolars) in plasma and lymph. It is generated from the metabolism of sphingomyelin through the actions of sphingomyelinase, ceramidase and sphingosine kinase (SPHK). Although platelets store high amounts of S1P, the main sources of plasma $\mathrm{S} 1 \mathrm{P}$ are erythrocytes ${ }^{13}$ and endothelial cells ${ }^{14}$, which exhibit high SPHK activity. S1P regulates many cellular functions via its interaction with the endothelial differentiation gene family of $G$ protein-coupled receptors, renamed as S1PR1-5 (ref. 15). S1P binds to its receptors within the low nanomolar range (the $K_{\mathrm{D}}$ of S1P for S1PRs ranges from 8 to $27 \mathrm{nM}$ ). S1PR activate different intracellular signalling pathways and differentially regulate endothelial cell function. S1PR1 couples to $G_{i}$ and activates the phosphatidylinositol-3-kinase (PI3K) pathway $^{16}$, which promotes vascular integrity ${ }^{17-19}$. In contrast, we found that S1PR2 antagonizes S1PR1-G $\mathrm{G}_{\mathrm{i}}-\mathrm{PI} 3 \mathrm{~K}$ signalling in the endothelium through activation of the $G_{12}$-Rho pathway ${ }^{20}$ and that S1PR2 is sufficient for endothelial activation in vitro ${ }^{21}$. The role of S1P in vascular development is well-illustrated by S1pr1 null mice, which exhibit a defect in vascular maturation ${ }^{18}$. In adult mice, S1PR1 promotes vascular integrity ${ }^{19}$ and regulates lymphocyte trafficking ${ }^{22}$. In fact, the recently FDA-approved Fingolimod, is a potent immunosuppressant via S1PR1. In contrast to S1PR1, S1PR2 is not required for embryonic vascular development and $S 1 \mathrm{pr}^{-1-}$ mice are viable and develop normally ${ }^{23}$. In addition, using a model of acute inflammatory vascular injury, we recently reported that S1PR2 expression in the stromal/endothelial compartment, and not in haematopoietic cells, is critical for the induction of vascular permeability, endothelial activation and sustained systemic inflammation ${ }^{21}$, suggesting that S1PR2 could be a novel target for acute vascular protection in various clinical settings.

Herein, we aimed to assess the role of S1PR2 in the regulation of cerebrovascular integrity after I/R injury. Using a mouse model of focal transient cerebral ischaemia, the middle cerebral artery occlusion model (MCAO), we report the critical role of S1PR2 in the disruption of cerebrovascular integrity after I/R injury. S1PR2 is induced in cerebral microvessels early after $I / R$ injury. Our in vitro studies with mouse and human primary brain microvascular endothelial cells (hBMVEC), mouse primary neurons and mouse primary mixed glial cells provide novel mechanistic insights into the regulation of cerebrovascular responses to $\mathrm{I} / \mathrm{R}$ injury by S1PR2. Finally, we detect S1PR2 in the cerebrovascular endothelium in human autopsy specimens. Altogether our data point to S1PR2 as a novel therapeutic target for acute cerebrovascular protection in stroke.

\section{Results}

Critical role of S1PR2 in neuronal injury in stroke. To study the role of S1PR2 signalling in the regulation of neurovascular responses to I/R injury, we used a mouse model of transient focal cerebral ischaemia, the transient MCAO (tMCAO) mouse model of stroke. S1pr2 ${ }^{+/+}$and S1pr2 ${ }^{-/-}$littermates were subjected to transient focal ischaemia for $90 \mathrm{~min}$. After $24 \mathrm{~h}$ of reperfusion, infarct and oedema ratios were quantified in wild-type and S1pr2 $2^{-/-}$mice as previously described ${ }^{24}$. As shown in Fig. 1a-c, S1pr2 ${ }^{-1}-$ mice exhibited a dramatic decrease in both infarct ratio (wild-type $0.324 \pm 0.053$ versus S1pr2 null $0.084 \pm 0.017$, $74 \%$ reduction, $P<0.05$, one-way analysis of variance (ANOVA) followed by Newman-Keuls test) and total cerebral oedema ratio (wild-type $0.121 \pm 0.011$ versus S1pr2 ${ }^{-/}-0.047 \pm 0.008,61.2 \%$ reduction, $P<0.05$, one-way ANOVA followed by NewmanKeuls test), which is the sum of cytotoxic and vasogenic oedema. In addition, S1pr $2^{-/-}$mice exhibited improved neurological scores compared with wild-type (Fig. 1d). We also found that the infarct size was significantly reduced and the neurological scores were significantly improved in S1pr2 null mice compared with wild-type, 5 days after tMCAO (Supplementary Fig. 1a-d).

To evaluate the therapeutic targeting of S1PR2 in stroke reperfusion therapies, we acutely inhibited S1PR2 activation by administration of the S1PR2 antagonist, JTE013 by gavage. In agreement with our data in S1pr2 null mice, administration of the S1PR2 antagonist $\left(30 \mathrm{mg} \mathrm{kg}^{-1}\right) 10 \mathrm{~min}$ after reperfusion $(1.7 \mathrm{~h}$ after the onset of ischaemia) in wild-type mice resulted in a dramatic decrease in both infarct and oedema ratios $(79.6 \%$ and 81.03\% inhibition, respectively, Fig. 1a-c), as well as in a significant improvement of the neurological scores (Fig. 1d). 
a

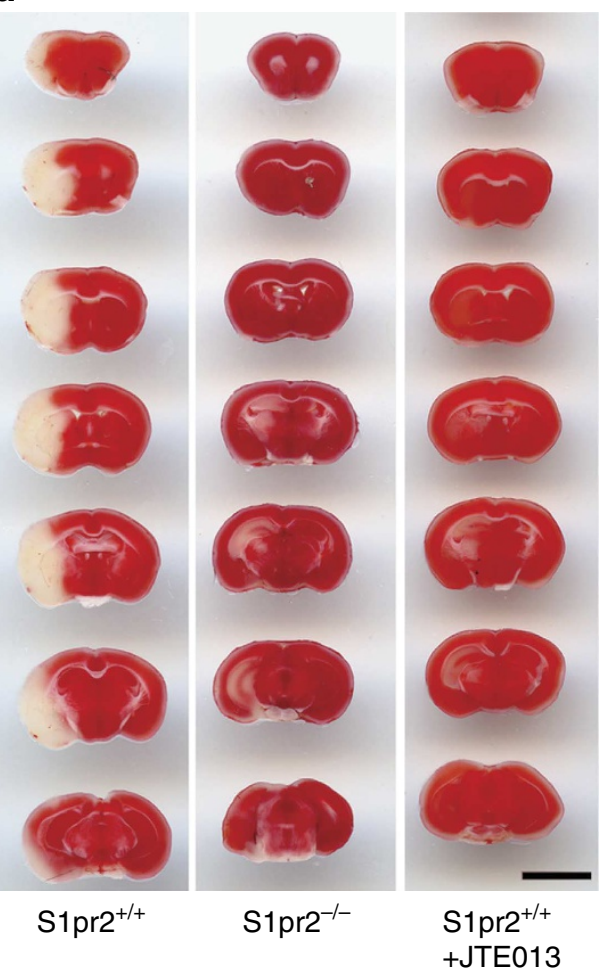

b
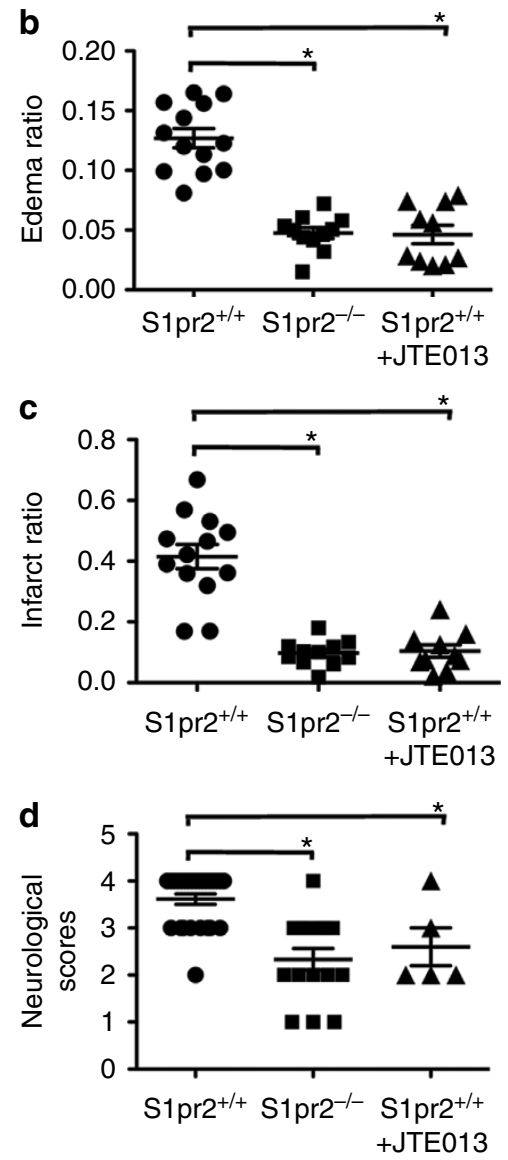

e
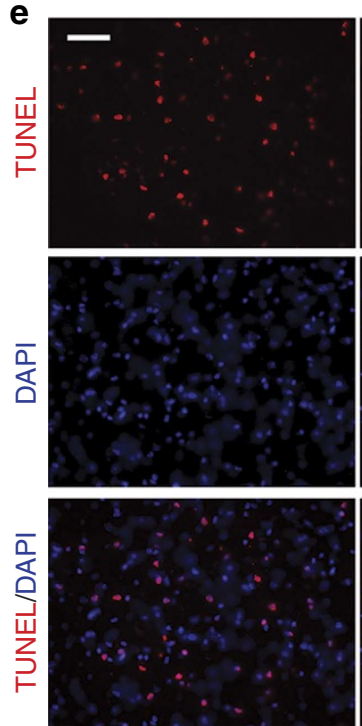

$\mathrm{S}_{\mathrm{ppr}}{ }^{+/+}$
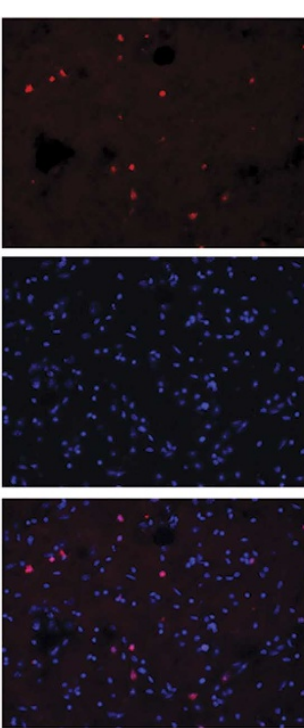

S1pr2-/-
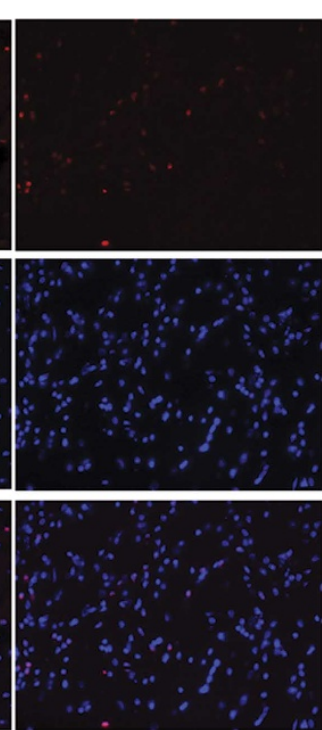

S1pr2 ${ }^{+/+}+$JTE013 f

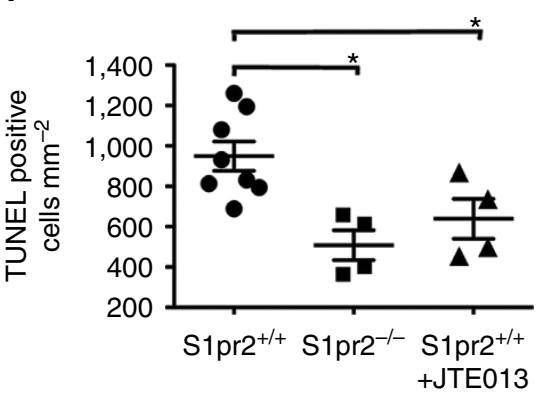

g

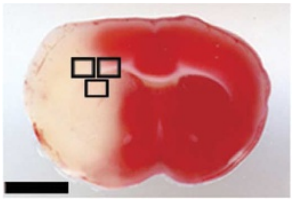

Figure 1 | Inhibition of S1PR2 results in less oedema and neuronal injury in experimental stroke. Ischaemia (90 min.) was induced in wild-type $\left(\mathrm{S} 1 \mathrm{pr} 2^{+/+}\right)$and S1pr2 ${ }^{-/-}$by MCAO. After reperfusion, mice received vehicle or the S1PR2 antagonist, JTE013 $\left(30 \mathrm{mg} \mathrm{kg}^{-1}\right)$, by gavage.

(a) Representative images of TTC staining of seven, 1-mm-thick brain coronal slices $24 \mathrm{~h}$ after reperfusion. Scale bar, $5 \mathrm{~mm}$. (b) Oedema and (c) infarct ratios were calculated by image analysis and reported as a ratio of the non-ischaemic hemisphere. Infarct ratios were corrected for oedema. The individual values and the mean \pm s.e.m. are shown. $N=10$-13 from eight independent experiments. ${ }^{\star} P<0.05$ (one-way ANOVA followed by Newman-Keuls).

(d) Improved neurological scores (that is, lower neurological deficit scores) were observed in S1pr ${ }^{-/-}$or JTE013-treated S1pr2 $+/+$mice compared with vehicle-treated S1pr2 $2^{+/}, 24 \mathrm{~h}$ after reperfusion. (e) Representative pictures of TUNEL assay (red channel) in the damaged area from S1pr2 ${ }^{+/+}$,

S1pr2 $2^{-/-}$and JTE013-treated S1pr2 ${ }^{+/+}$mice. Blue channel: nuclear staining (DAPI). (f) Quantification of TUNEL-positive cells per mm². Values are the average of TUNEL-positive cells from three fields. $n=4-8$ from four independent experiments. Scale bar, $50 \mu \mathrm{m}$. (g) Image showing the anatomical areas in which TUNEL-positive cells were quantified in mice (bregma +1.2 to $+0.8 \mathrm{~mm}$, rostrally). Scale bar, $2.5 \mathrm{~mm}$. 
Consistent with our recent studies using sepsis models ${ }^{21}$, the minimum effective dose of JTE013 was $30 \mathrm{mg} \mathrm{kg}^{-1}$ (Supplementary Fig. 2). Mortality was $<5 \%$ in all cohorts of mice $24 \mathrm{~h}$ after reperfusion.

Terminal deoxynucleotidyl transferase dUTP nick end labelling (TUNEL) assays showed a significant decrease in TUNELpositive cells both in S1pr2 $2^{-/-}$and JTE013-treated S1pr2 $2^{+/+}$ mice $\left(508.7 \pm 74\right.$ and $639 \pm 98$ positive cells per $\mathrm{mm}^{2}$, respectively) compared with vehicle-treated wild-type mice $(949 \pm 73$ positive cells per $\mathrm{mm}^{2}$ ) (Fig. 1e,f), indicating that inhibition of S1PR2 decreases DNA fragmentation that results from apoptosis after I/R injury. No TUNEL-positive cells were detected in the contralateral hemisphere (not shown).

No significant differences were observed in the physiological parameters (arterial $\mathrm{O}_{2}$ saturation, heart rate, pulse distention and respiratory rate) between wild-type and $S 1 \mathrm{pr}^{-/-}$mice, before, during or after MCAO (Table 1). In addition, cerebral blood flow $(\mathrm{CBF})$ in the territory of the MCA, monitored during the surgeries by Laser Doppler, was similarly reduced in all three groups of mice during occlusion $(13 \% \pm 1.2$ of basal level) and similarly restored after reperfusion in wild-type, $S 1 \mathrm{pr}^{-/-}$and JTE013-treated wild-type mice ( $94.6 \% \pm 1.1$ of basal) (Fig. 2).

To determine the therapeutic time window of JTE013 in experimental stroke, we assessed the oedema and infarct ratios after delayed administration of the S1PR2 antagonist. We found that JTE013 administration $4.5 \mathrm{~h}$ after ischaemia resulted in the same significant reduction of brain infarct and oedema ratios compared with $1.7 \mathrm{~h}$ or S1pr2 $2^{-/-}$groups (no statistically significant differences were found between the $4.5 \mathrm{~h}, 1.7 \mathrm{~h}$ and S1pr $2^{-/}$groups, Supplementary Fig. 3), indicating that JTE013 was efficacious in reducing brain infarct and oedema when administered within $4.5 \mathrm{~h}$ after stroke. Administration of JTE013 at $7.5 \mathrm{~h}$ after the onset of ischaemia had reduced efficacy and did not lead to statistically significant lower infarct sizes compared with the vehicle-treated group, indicating that JTE013 is protective when given within $4.5 \mathrm{~h}$ of stroke onset.

Altogether our data indicate that genetic deletion of S1PR2 or administration of a S1PR2 antagonist in wild-type mice after reperfusion, dramatically decreased infarct size, total oedema and cell death in experimental stroke, resulting in improved neurological scores, indicating that S1PR2 could be a novel therapeutic target in stroke.

Critical role of S1PR2 in cerebrovascular injury. During cerebral ischaemia, blood-brain barrier disruption starts early after the onset of ischaemia and increased cerebrovascular permeability can be detected as early as $3 \mathrm{~h}$ after reperfusion ${ }^{25,26}$. To investigate the role of S1PR2 in vasogenic oedema (increased cerebrovascular permeability), we conducted Evans blue dye (EBD) extravasation assays. As shown in Fig. 3a,b, S1pr2-1mice exhibited a dramatic decrease in EBD extravasation, $3 \mathrm{~h}$ after reperfusion, compared with their wild-type litter mates (ratio of EBD content in ipsilateral hemisphere/contralateral hemisphere was $1.6 \pm 0.31$ in $S 1 p r 2^{-1-}$ mice versus $4.54 \pm 0.83$ in wild-type). In addition, acute inhibition of S1PR2 signalling in wild-type mice, by JTE013 administration, potently blocked the induction of cerebrovascular permeability after I/R injury (ratio of EBD content $1.28 \pm 0.08$, Fig. 3a,b).

Increasing clinical evidence indicates that areas of the brain with compromised blood-brain barrier at the early stages of ischaemic stroke often undergo haemorrhagic transformation following reperfusion ${ }^{27-30}$. To study the role of S1PR2 in the development of intracerebral haemorrhage after I/R injury, we used a model of spontaneous haemorrhagic transformation. Mice were subjected to $3 \mathrm{~h}$ of ischaemia to induce severe neurovascular injury. On this prolonged time of ischaemia, a significant loss of cerebrovascular integrity was observed $24 \mathrm{~h}$ after reperfusion in wild-type mice (Fig. 3c,d), as assessed by the presence of extravascular blood in the ischaemic hemisphere $(448 \pm 96.4 \mathrm{mg}$ haemoglobin) compared with the contralateral hemisphere (182 $\pm 23.9 \mathrm{mg}$ haemoglobin/hemisphere). The ipsilateral/ contralateral ratio was $2.68 \pm 0.74$ in wild-type mice (Fig. $3 \mathrm{~d}$ ). In sharp contrast, intracerebral haemorrhage was almost absent in $S 1$ pr2 ${ }^{-1-}$ mice (ipsilateral/contralateral ratio $1.01 \pm 0.17$, $\sim 80 \%$ inhibition). In addition, acute inhibition of S1PR2 by oral administration of JTE013, 10 min after reperfusion, dramatically inhibited the development of intracerebral haemorrhage (ipsilateral/contralateral ratio $1.04 \pm 0.05$ ). The mortality rate at $24 \mathrm{~h}$ after a 3 -h occlusion was $39 \%$ in vehicle-treated wild-type mice, $20 \%$ in $S 1 p r 2^{-1-}$ and $18.2 \%$ in wild-type mice treated with JTE013.

\begin{tabular}{|c|c|c|c|}
\hline Time points & Parameters & S1pr2 $+/+$ & S1pr2-/- \\
\hline \multirow[t]{4}{*}{$\begin{array}{l}10 \text { min before } \\
M C A O\end{array}$} & $\begin{array}{l}\text { Arterial } \mathrm{O}_{2} \text { saturation } \\
\left(\mathrm{S}_{\mathrm{p}} \mathrm{O}_{2}, \%\right)\end{array}$ & $97.0 \pm 0.6$ & $98.4 \pm 0.2$ \\
\hline & Heart rate (b.p.m.) & $493.0 \pm 9.2$ & $520.9 \pm 31$ \\
\hline & Pulse distention $(\mu \mathrm{m})$ & $13.4 \pm 4.5$ & $18.3 \pm 8.3$ \\
\hline & $\begin{array}{l}\text { Respiratory rate } \\
\text { (br.p.m.) }\end{array}$ & $110.1 \pm 11.1$ & $109.1 \pm 5.1$ \\
\hline \multirow[t]{4}{*}{$\begin{array}{l}10 \text { min after } \\
\text { MCAO }\end{array}$} & $\begin{array}{l}\text { Arterial } \mathrm{O}_{2} \text { saturation } \\
\left(\mathrm{S}_{\mathrm{p}} \mathrm{O}_{2}, \%\right.\end{array}$ & $97.0 \pm 0.6$ & $98.2 \pm 0.3$ \\
\hline & Heart rate (b.p.m.) & $531.3 \pm 13.8$ & $524 \pm 13.5$ \\
\hline & Pulse distention $(\mu \mathrm{m})$ & $19.3 \pm 5.8$ & $15.7 \pm 3.7$ \\
\hline & $\begin{array}{l}\text { Respiratory Rate } \\
\text { (br.p.m.) }\end{array}$ & $104.5 \pm 3.5$ & $115.7 \pm 8.3$ \\
\hline \multirow[t]{4}{*}{$\begin{array}{l}10 \text { min after } \\
\text { reperfusion }\end{array}$} & $\begin{array}{l}\text { Arterial } \mathrm{O}_{2} \text { saturation } \\
\left(\mathrm{S}_{\mathrm{p}} \mathrm{O}_{2}, \%\right)\end{array}$ & $96.8 \pm 1.1$ & $98.1 \pm 0.2$ \\
\hline & Heart rate (b.p.m.) & $496.6 \pm 14.76$ & $525.6 \pm 11$ \\
\hline & Pulse distention $(\mu \mathrm{m})$ & $16.6 \pm 1$ & $13.0 \pm 3.3$ \\
\hline & $\begin{array}{l}\text { Respiratory rate } \\
\text { (br.p.m.) }\end{array}$ & $107.8 \pm 5.8$ & $112.2 \pm 5.2$ \\
\hline
\end{tabular}

bpm, beats per minute; br.p.m., breaths per minute; MCAO, middle cerebral artery occlusion. Pulse distention is a surrogate for pulse pressure (pulse pressure $=$ systolic pressure - diastolic pressure). JTE013 (30 $\mathrm{mg} \mathrm{kg}^{-1}$ ) or vehicle was administered after reperfusion. No significant differences were observed in the physiological parameters (arterial $\mathrm{O}_{2}$ saturation, heart rate, pulse distention and respiratory rate) between wild-type and S1pr $2^{-/-}$mice, before, during or after MCAO.

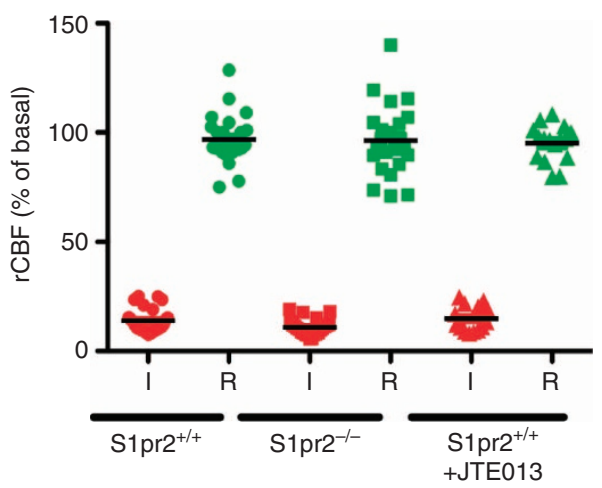

Figure 2 | Regional cerebral blood flow (CBF) in S1pr2 ${ }^{+/+}$and S1pr2 ${ }^{-1-}$ mice. Regional CBF in the middle cerebral artery territory was measured throughout the surgeries using a laser Doppler probe, attached to the temporal bone. The relative CBF (\% of basal) during MCA occlusion ( $\mathrm{I}$, red circles, squares and triangles) and $10 \mathrm{~min}$ after reperfusion ( $\mathrm{R}$, green

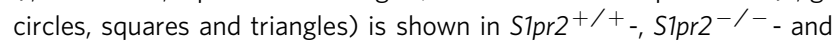
S1pr2 $2^{+/+}$-receiving JTEO13 is shown. $N=18-30$. 
a

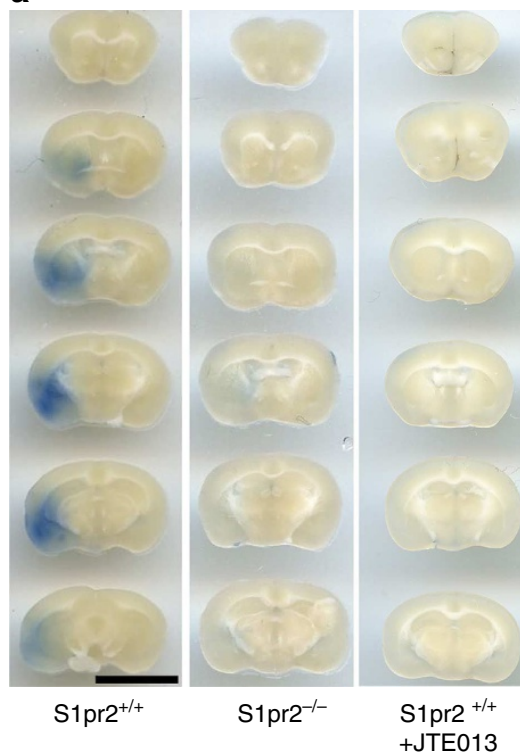

b

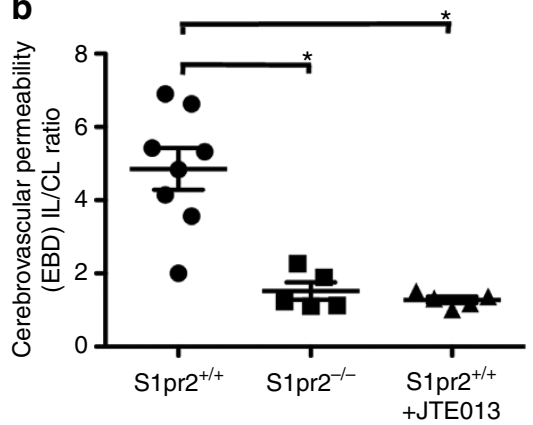

C

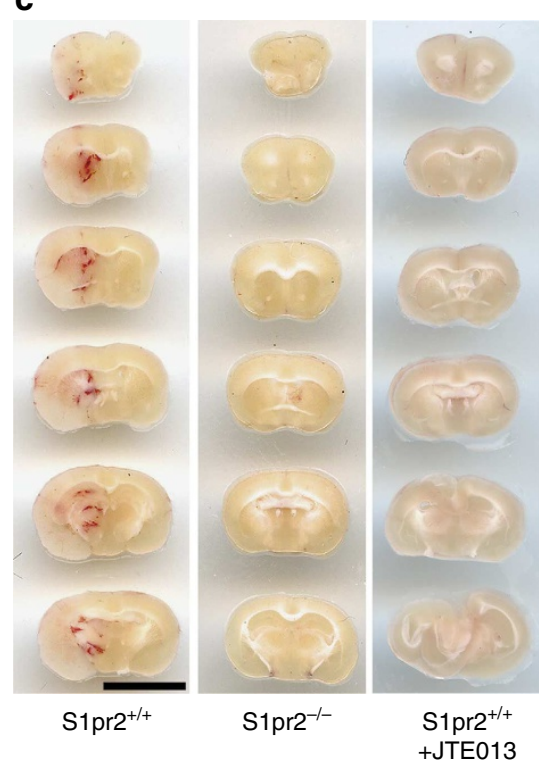

d

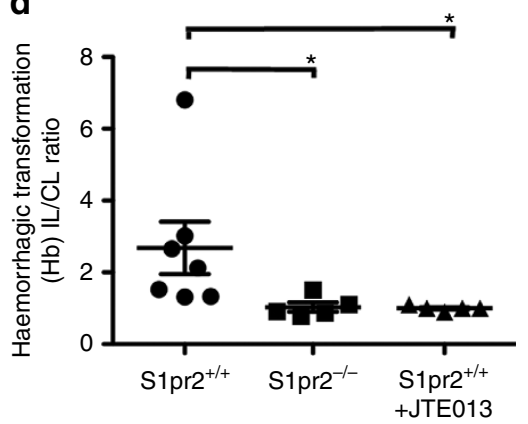

Figure 3 | Critical role of S1PR2 in the disruption of cerebrovascular integrity after I/R injury. (a,b) Cerebrovascular permeability (vasogenic oedema) was determined by Evans blue dye (EBD) extravasation assay, $3 \mathrm{~h}$ after reperfusion in S1pr2 ${ }^{+/+}$, S1pr2 ${ }^{-/-}$and S1pr2 ${ }^{+/+}$receiving JTE013. (a) Brain coronal slices of representative animals. Scale bar, $5 \mathrm{~mm}$. (b) Quantification of extravascular EBD. Ipsilateral/contralateral (IL/CL) ratios are shown. (c,d) Haemorrhagic transformation was assessed $24 \mathrm{~h}$ after reperfusion in S1pr2 ${ }^{+/+}$, S1pr2 ${ }^{-/-}$and S1pr2 ${ }^{+/+}$receiving JTE013. (c) Representative images are shown. Scale bar, $5 \mathrm{~mm}$. (d) Quantification of extravascular haemoglobin $(\mathrm{Hb})$ content. Values are ipsilateral/contralateral $(\mathrm{IL} / \mathrm{CL})$ ratios of micrograms of $\mathrm{Hb}$. (b,d) The individual values and the mean \pm s.e.m. are shown, $n=5-8$ from four independent experiments. ${ }^{\star} P<0.05$ (one-way ANOVA followed by Newman-Keuls).

These data indicate the critical role of S1PR2 in the disruption of cerebrovascular integrity after $I / R$ injury in the brain, suggesting that S1PR2 could be targeted in stroke patients at the time of reperfusion to reduce cerebrovascular permeability and haemorrhagic transformation.

Critical role of S1PR2 in MMP-9 activation after stroke. To elucidate the mechanisms of regulation of cerebrovascular integrity by S1PR2, we determined the activation of MMP-2 (gelatinase A) and MMP-9 (gelatinase B), also known as type IV collagenases, since they play a critical role in increased cerebrovascular permeability after $I / R$ injury ${ }^{31,32}$. In wild-type animals, MMP-9 activity was significantly increased in brain lysates $3 \mathrm{~h}$ after $\mathrm{I} / \mathrm{R}$ injury, compared with sham animals (Fig. 4a,b). Interestingly, S1pr2 $2^{-1-}$ mice exhibited significantly decreased MMP-9 activity compared with wild-type (56 $\pm 10 \%$ inhibition). Similarly, in JTE013-treated wild-type mice, MMP-9 activity was significantly lower compared with vehicle-treated wild-type mice ( $57 \pm 6.9 \%$ inhibition). In contrast, we did not detect a significant increase in MMP-2 activity in total brain lysates after I/R compared with sham animals or differences in
MMP-2 activity between wild-type and $S 1 p r 2^{-/-}$mice or JTE013-treated mice $3 \mathrm{~h}$ after reperfusion. These data indicate that S1PR2 plays a critical role in the induction of MMP-9 activity in brain lysates after $\mathrm{I} / \mathrm{R}$ injury. In situ zymography assays revealed a dramatic increase in gelatinase activity both in cerebral microvessels and in brain parenchyma in the damaged area in vehicle-treated wild-type mice, $3 \mathrm{~h}$ after reperfusion (Fig. 4c) (no signal was detected in sham animals or in the contralateral hemisphere). Interestingly, S1pr2 ${ }^{-1-}$ and JTE013-treated S1pr2 ${ }^{+/+}$mice exhibited much lower gelatinase activity in cerebral microvessels, consistent with the decreased cerebrovascular integrity observed in the EBD cerebrovascular permeability assay. Representative pictures of the damaged area at the level of bregma $+1 \mathrm{~mm}$ (rostrally) are shown, as indicated in Fig. 1g. Altogether these data indicate that S1PR2 plays a critical role in MMP-9 activation and the induction of gelatinase activity in cerebral microvessels after $\mathrm{I} / \mathrm{R}$ injury in experimental stroke.

Detection of S1PR2 in cerebral microvessels after $I / R$ injury. We next aimed to assess the expression of S1PR2 in the mouse 

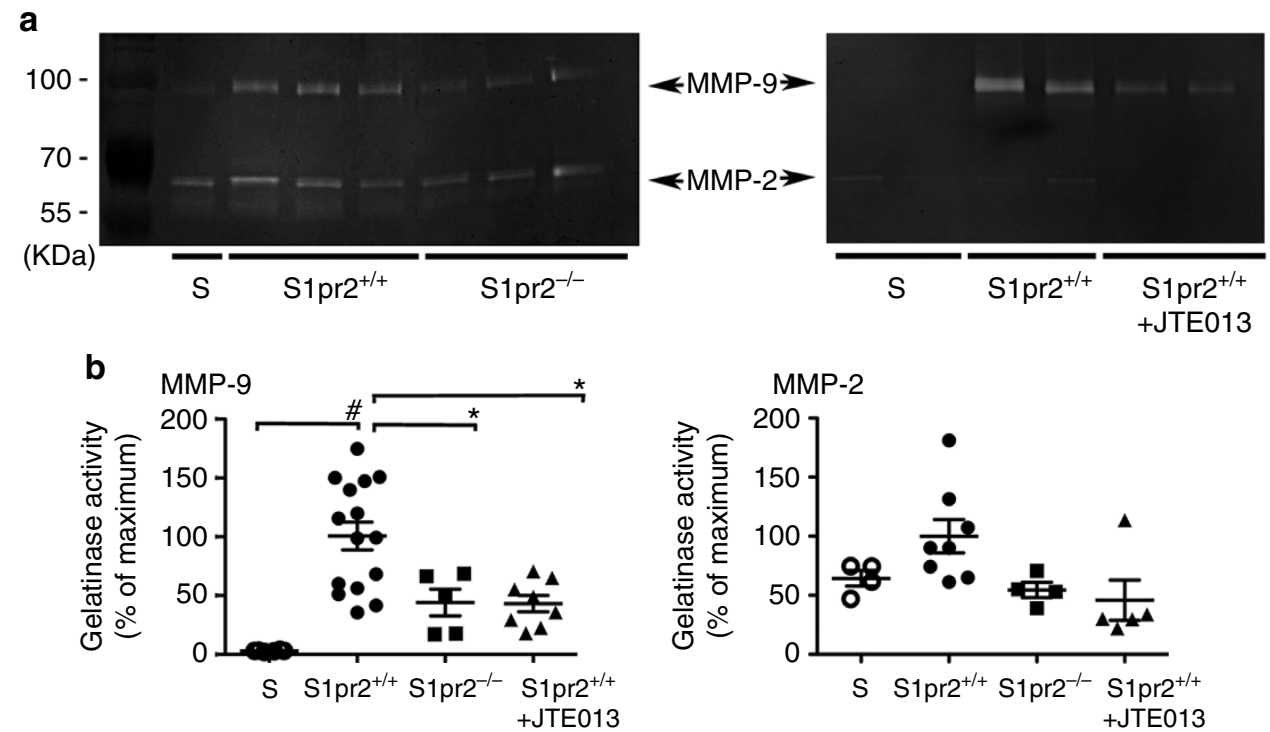

C

$\mathrm{S}_{\mathrm{pr}} \mathrm{pr}^{+/+}$

$\mathrm{S}_{\mathrm{p} p 2^{-1-}}$

S1pr2 ${ }^{+/+}+$JTE013

듬
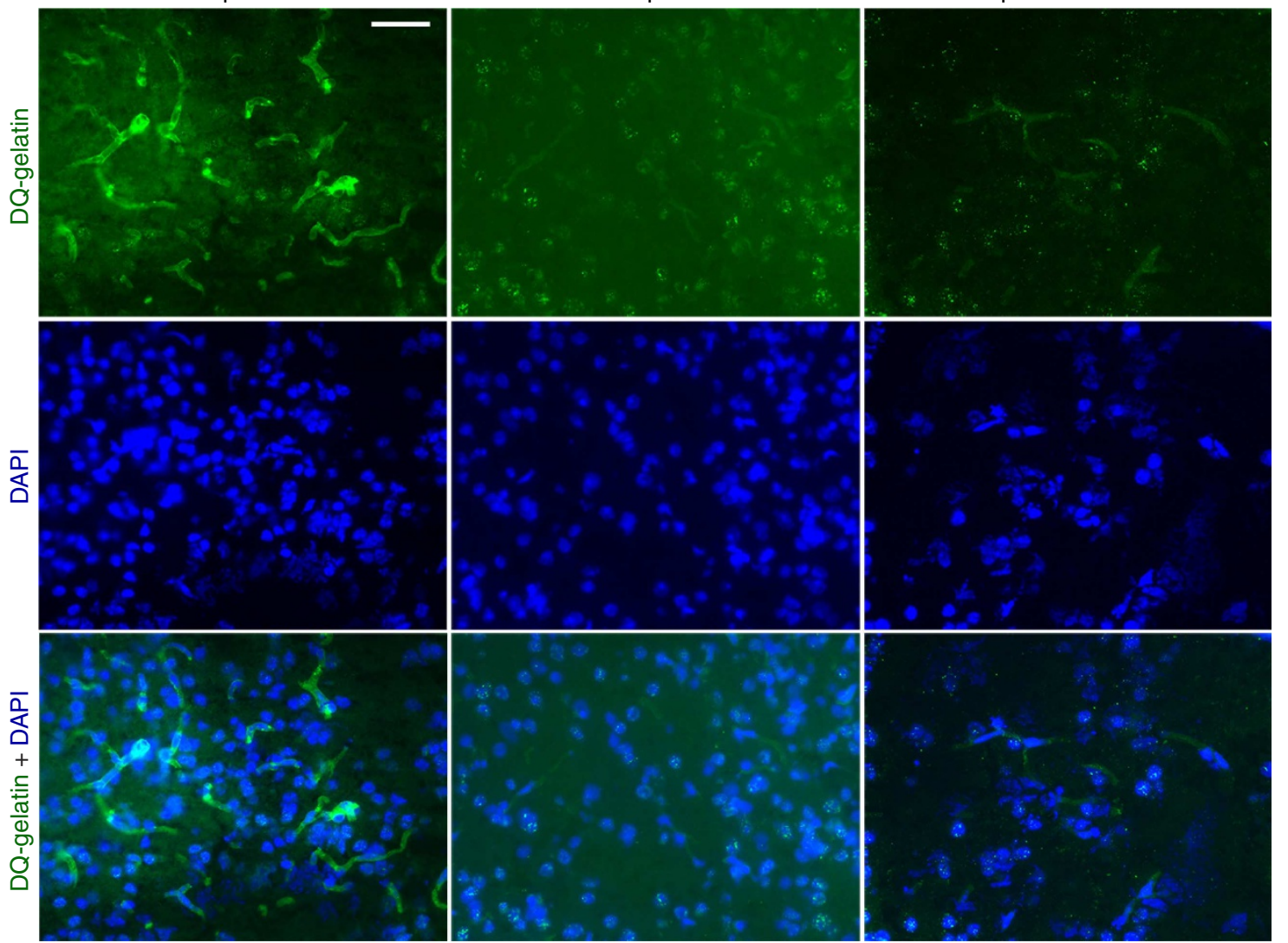

Figure 4 | Blockade of S1PR2 results in decreased MMP-9 activity in ischaemic brains. (a) Representative zymographies showing MMP-9 and MMP-2 activity, $3 \mathrm{~h}$ after I/R in whole brain homogenates, in sham (S), vehicle-treated S1pr2 ${ }^{+/+}\left(\right.$S1pr2 $\left.{ }^{+/+}\right)$, vehicle-treated S1pr2 ${ }^{-/-}\left(\right.$S1pr2 $\left.{ }^{-/-}\right)$and JTE013treated S1pr2 $+/+$ mice (S1pr2 $+/++$ JTE013). Arrows indicate active MMP-9 and MMP-2. (b) Quantification of gelatinase activity by image analysis. Values are normalized by the average gelatinase activity in wild-type mice after $\mathrm{I} / \mathrm{R}$ injury. $\mathrm{N}=4-15$ from five independent experiments. $\# P<0.05$ sham versus $\mathrm{MCAO},{ }^{\star} P \leq 0.05$ vehicle-treated S1pr2 ${ }^{+/+}$versus JTE013-treated S1pr2 ${ }^{+/+}$or vehicle-treated S1pr2 ${ }^{-/-}$(one-way ANOVA followed by Newman-Keuls). (c) Representative pictures of the damaged area (at the level of bregma +1.2 to $+0.8 \mathrm{~mm}$, rostrally, as indicated in Fig .1g) of the in situ gelatinase assay in frozen brain sections $(14 \mu \mathrm{m})$ using FITC DQ-gelatin are shown. Notice significantly lower gelatinase activity in brain microvessels in vehicle-treated S1pr2 ${ }^{-/-}$or JTE013-treated S1pr2 ${ }^{+/+}$mice compared with vehicle-treated wild-type, $3 \mathrm{~h}$ after reperfusion. $\mathrm{N}=5-6 \mathrm{per}$ group, 4 independent experiments. Scale bar, $50 \mu \mathrm{m}$.

brain after I/R injury. In total brain lysates, we found that S1PR2 mRNA basal levels were low $\left(0.37 \pm 0.03\right.$ mRNA copies $/ 10^{6} 18 \mathrm{~S}$ mRNA) but were significantly increased in the ischaemic hemisphere after I/R injury (2.6 \pm 0.37 - and $3.1 \pm 0.35$-fold at 6 and $24 \mathrm{~h}$, respectively, Fig. 5a). Consistent with this mRNA data, we detected S1PR2 protein $6 \mathrm{~h}$ after I/R injury by 


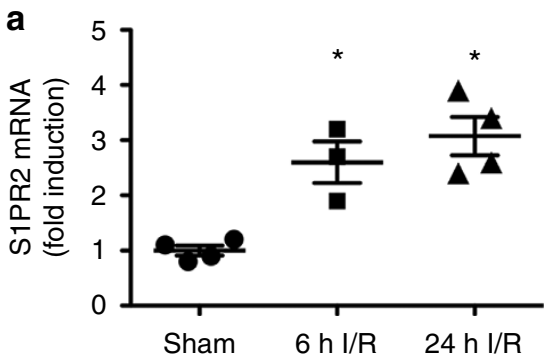

b
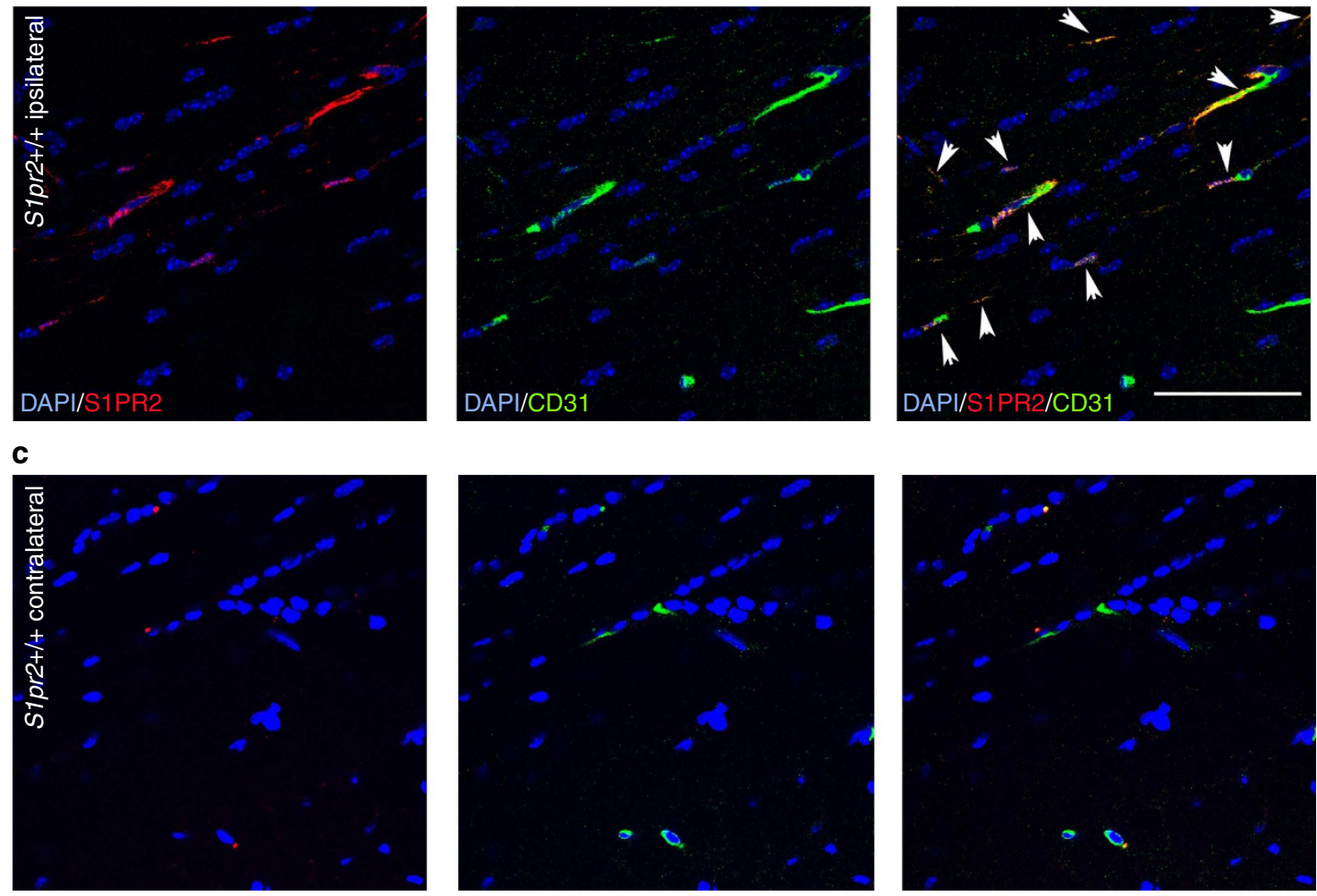

d
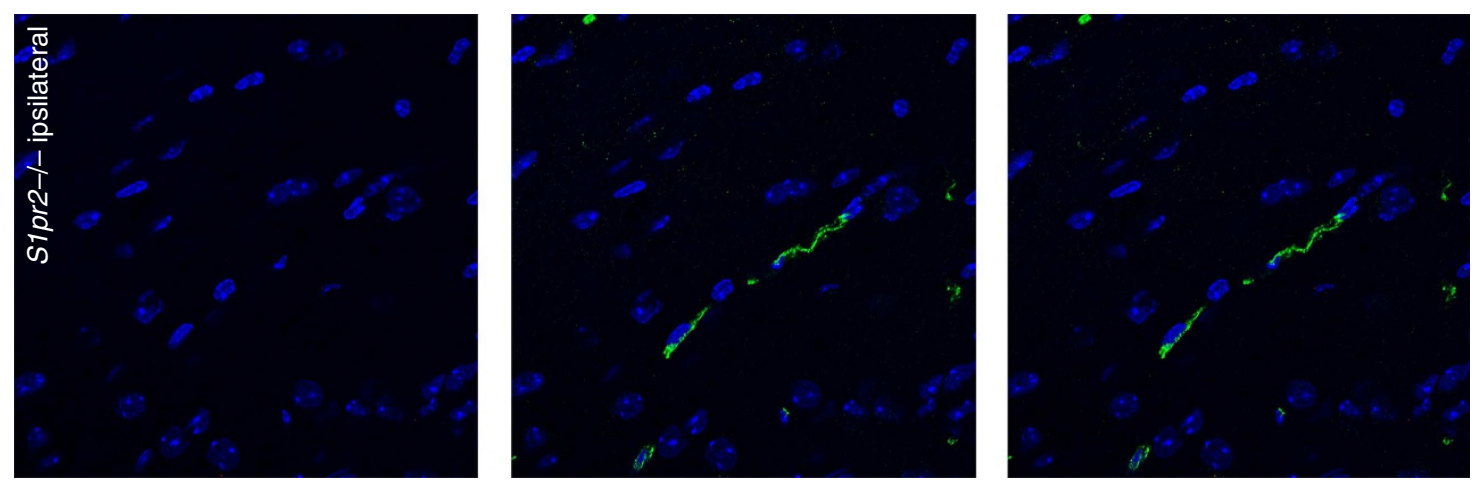

Figure 5 | Upregulation of S1PR2 in brain microvessels after I/R injury. (a) S1PR2 mRNA levels in the ipsilateral hemisphere of brain homogenates from sham animals and MCAO animals, $6 \mathrm{~h}(6 \mathrm{~h} \mathrm{I} / \mathrm{R})$ or $24 \mathrm{~h}(24 \mathrm{~h} \mathrm{I} / \mathrm{R})$ after tMCAO. The fold induction of S1PR2 mRNA levels (normalized by $18 \mathrm{~S}$ RNA) versus sham is shown. ${ }^{\star} P<0.05$ (one-way ANOVA followed by Newman-Keuls). (b-d) S1PR2 (red channel) and CD31 (green channel) immunofluorescence analysis in brain sections from the ipsilateral (b) or contralateral (c) hemisphere of wild-type mice or ipsilateral hemisphere of S1pr2 $2^{-/-}$mice (d) $6 \mathrm{~h}$ after tMCAO. Representative pictures of the corpus callosum at the level of bregma +1.2 to $+0.8 \mathrm{~mm}$, rostrally, as indicated in Fig. $1 \mathrm{~g}$, are shown ( $n=5-6$ per group). S1PR2 was detected only in cerebral microvessels in the damaged area (ipsilateral hemisphere), (b) but not in the contralateral hemisphere (c), the ipsilateral hemisphere of S1pr2-/- mice (d), or in sham animals (not shown). Arrows indicate the S1PR2 and CD31 positive areas. Scale bar, $50 \mu$ m.

immunofluorescence analysis only in the ischaemic hemisphere of wild type mice (Fig. 5b). Representative pictures of the damaged area (corpus callosum and striatum) at the level of bregma
$+1 \mathrm{~mm}$ (as indicated in Fig. 1g) are shown (Fig. 5b and Supplementary Fig. 4a, respectively). S1PR2 positivity colocalized with CD31 staining (Fig. 5b and Supplementary Fig. 4a). No 

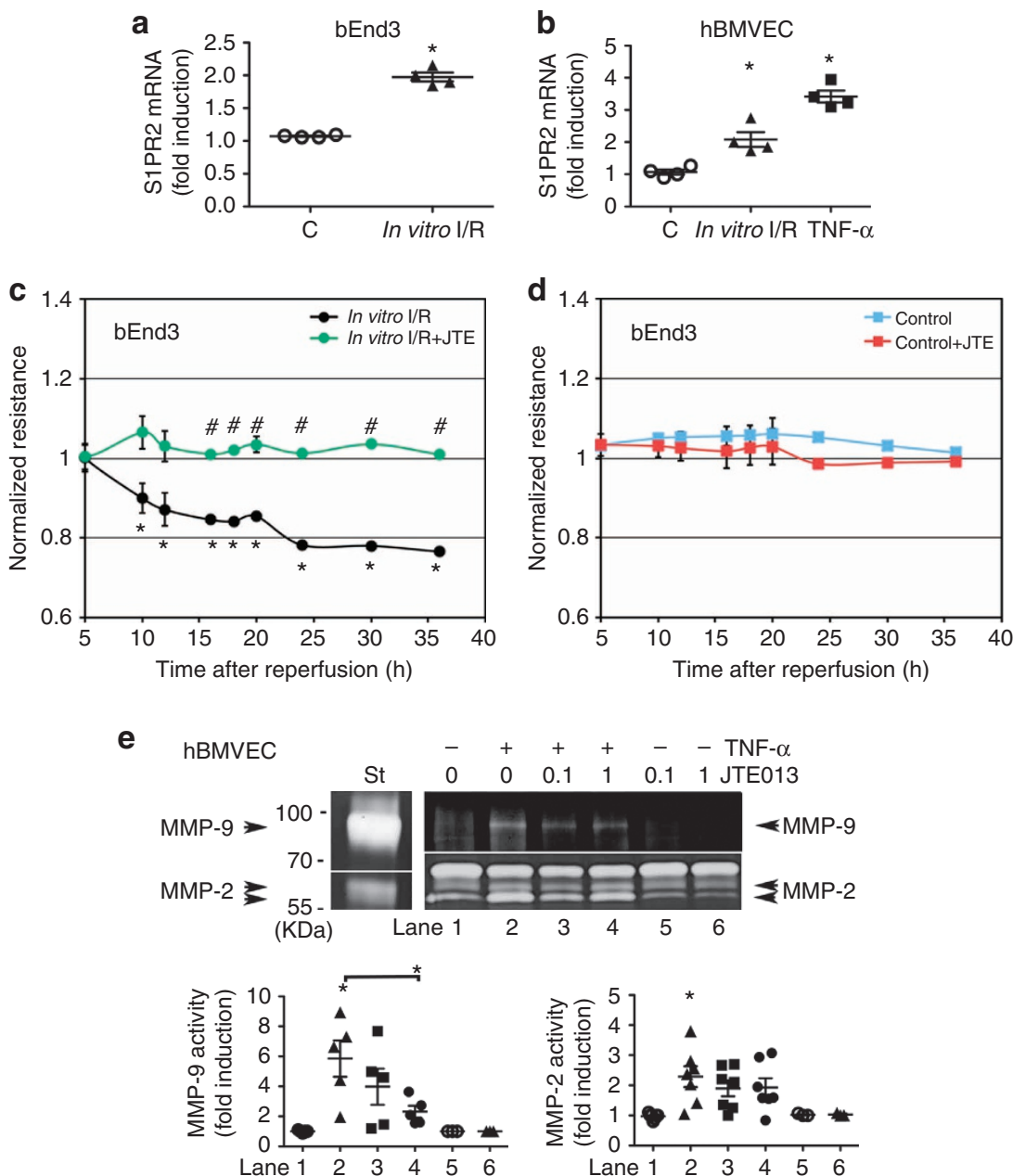

Figure 6 | Critical role of S1PR2 in brain endothelial cell permeability and MMP-9 activation. (a,b) Induction of S1PR2 mRNA in bEnd.3 and hBMVEC after in vitro I/R injury or activation with TNF- $\alpha$. Brain endothelial cells were subjected to (a) $6 \mathrm{~h}$ of OGD followed by in vitro reperfusion or (b) activation with TNF- $\alpha\left(5 \mathrm{ng} \mathrm{ml}^{-1}\right) .6 \mathrm{~h}$ later, RNA was isolated and S1PR2 mRNA levels were quantified by qRT-PCR analysis. Fold induction of S1PR2 mRNA (normalized by $18 \mathrm{~S}$ RNA) versus control (C) is shown. ${ }^{\star} P \leq 0.05$ in vitro I/R or TNF- $\alpha$ versus control (Student's $t$-test). Values are mean \pm s.e.m., $n=4$. (c,d) S1PR2 inhibition by JTE013 prevented the decrease in monolayer resistance in bEnd.3 cells after in vitro I/R injury. (c) In vitro I/R. (d) Control (normoxia). Values are mean \pm s.e.m. $n=3-7$ from three independent experiments. ${ }^{\star} P \leq 0.05$ in vitro $\mathrm{I} / \mathrm{R}$ versus control (normoxia, right panel), $\# P \leq 0.05$ JTE013-treated in vitro I/R versus vehicle-treated in vitro I/R (Student's $t$-test). Black: in vitro I/R + vehicle, green: in vitro I/R + JTE013, blue: control (normoxia) + vehicle, red: control (normoxia) + JTE013. (e) MMP-9 and MMP-2 activity in human brain microvascular endothelial cell (hBMVEC) supernatants after treatment with $5 \mathrm{ng} \mathrm{ml}^{-1} \mathrm{TNF}-\alpha$ in the presence or absence of the S1PR2 antagonist JTE013 $(0.1$ or $1 \mu \mathrm{M})$ for $24 \mathrm{~h}$. Values are mean \pm s.e.m. $n=4-5$ from four independent experiments. A representative zymography is shown. ${ }^{\star} P \leq 0.05$ TNF- $\alpha$ versus control, and when indicated TNF- $\alpha+$ vehicle versus TNF- $\alpha+$ JTE013-treated (one-way ANOVA followed by Newman-Keuls). St: active MMP-2 and MMP-9 standards. Arrows indicate active MMP-9 and MMP-2.

S1PR2 immunopositivity was detected in the contralateral hemisphere of wild type mice $6 \mathrm{~h}$ after I/R injury (Fig. $5 \mathrm{c}$ and Supplementary Fig. 4b), in the ispilateral hemisphere of $S 1$ pr2 ${ }^{-/}$ - mice (Fig. 5d and Supplementary Fig. 4c) or in sham animals. These data indicate that S1PR2 upregulation in cerebral microvessels is an early event after I/R injury.

S1PR2 is critical for brain endothelial cell activation. Given our in vivo findings in mice, we next aimed to study the role of S1PR2 in the regulation of the responses of brain endothelial cells to I/R injury in vitro. We used the mouse brain microvascular endothelial cell line, bEnd3 (ref. 33), and hBMVEC. To mimic in vitro the metabolic and inflammatory stress of $I / R$ injury, we conducted oxygen and glucose deprivation (OGD) experiments (in vitro $\mathrm{I} / \mathrm{R}$ ), as well as $\mathrm{TNF}-\alpha$ activation studies. In vitro $\mathrm{I} / \mathrm{R}$ injury or TNF- $\alpha$ activation resulted in upregulation of S1PR2 message (2- and 3.5-fold, respectively) both in bEnd3 and hBMVEC (Fig. 6a,b). To study the role of S1PR2 in brain endothelial cell permeability, bEnd3 cells were exposed to hypoxia/aglycemia (OGD) for $6 \mathrm{~h}$. Then, we measured changes in endothelial resistance on in vitro reperfusion, using the electrical cell-substrate impedance sensing system (ECIS) (Applied BioPhysics, NY $)^{34}$. As shown in Fig. 6c, in vehicle-treated cells, endothelial resistance starts to decrease $10 \mathrm{~h}$ after in vitro reperfusion and remains lower compared with control (normoxia, Fig. 6d). However, inhibition of S1PR2 signalling by JTE013 abrogated the decrease in monolayer resistance observed after in vitro I/R injury (Fig. 6c), indicating the critical role of S1PR2 in the induction of brain endothelial cell monolayer permeability after in vitro I/R injury. JTE013 treatment did not affect endothelial resistance under normoxic conditions (Fig. 6d). These experiments were conducted in the presence of serum, which contains S1P. In addition, since endothelial cells exhibit 

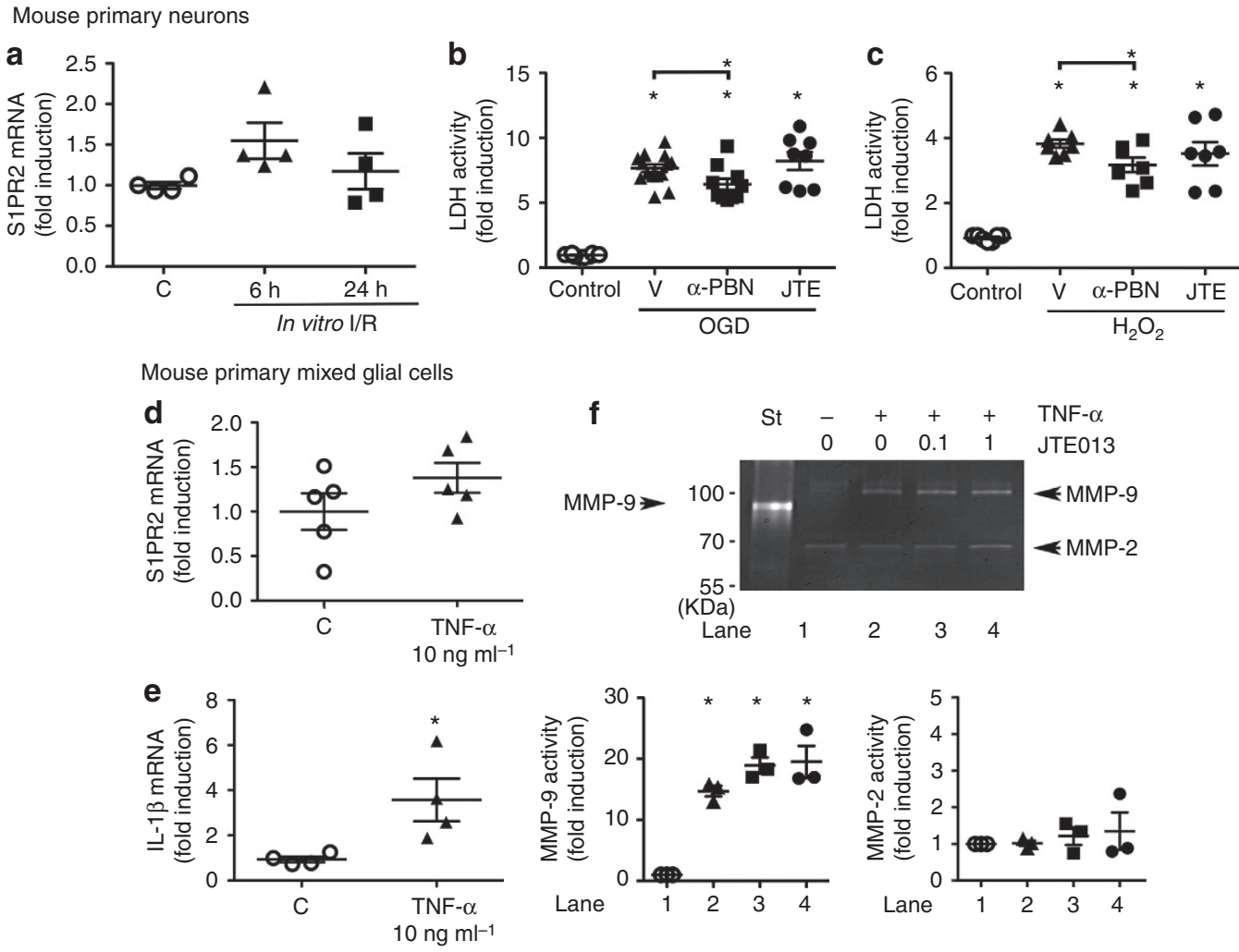

Figure 7 | Role of S1PR2 in the responses of neurons and glial cells to injury. Mouse primary neurons (a-c) and mouse primary mixed glial cells (d,e) were isolated and cultured as described in Methods. (a) Mouse primary neurons were subjected to $4 \mathrm{~h}$ of OGD. 6 or $24 \mathrm{~h}$ after in vitro reperfusion, RNA was isolated and S1PR2 mRNA copy number was calculated by qRT-PCR analysis and normalized by $10^{6}$ copies of $18 \mathrm{~S}$ RNA. Fold induction versus control ( $C$; normoxia, normoglycemia) is plotted. (b) Mouse primary neurons were subjected to $4 \mathrm{~h}$ of OGD. On in vitro reperfusion, cells were treated with vehicle (V), $\alpha$-PBN $(1 \mathrm{mM})$ or JTEO13 $(1 \mu \mathrm{M})$ for $24 \mathrm{~h}$. Neuronal cell death was assessed by measuring LDH activity in the supernatants. (c) Primary neurons were treated with $\mathrm{H}_{2} \mathrm{O}_{2}(30 \mu \mathrm{M})$ for $24 \mathrm{~h}$ in the presence or absence of $1 \mathrm{mM} \alpha$-PBN or $1 \mu \mathrm{M}$ JTE 013 (JTE) and LDH activity was measured. Control: no $\mathrm{H}_{2} \mathrm{O}_{2}$. V: vehicle. (d,e) Mouse primary mixed glial cells were activated with $5 \mathrm{ng} \mathrm{ml}^{-1} \mathrm{TNF}-\alpha$ to mimic the pro-inflammatory environment of I/R injury. $24 \mathrm{~h}$ later, S1PR2 (d) and IL-1 $\beta$ (e) mRNA levels were assessed by qRT-PCR analysis. (f) Mouse primary mixed glial cells were activated with $5 \mathrm{ng} \mathrm{ml}^{-1}$ TNF- $\alpha$ in the presence or absence (vehicle) of JTE013 at the doses indicated $(\mu \mathrm{M})$. MMP-9 and MMP-2 activity in the conditioned media was assessed by gelatin zymography. Values are mean \pm s.e.m. $n=3$ from three independent experiments. A representative zymography is shown. ${ }^{\star} P \leq 0.05$ TNF- $\alpha$ versus control, and when indicated TNF- $\alpha$ versus TNF- $\alpha$ + JTE013 treated (one-way ANOVA followed by Newman-Keuls). St: active MMP-9 standard. (a-e) Fold induction versus control is plotted.

high levels of SPHK activity ${ }^{19}$ and are one of the main sources of plasma S1P ${ }^{14}$ it is possible that endothelial cell-derived S1P activates S1PR in an autocrine way, as we have previously described $^{20}$.

Given that our in vitro model demonstrated similar S1PR2mediated vascular responses to $\mathrm{I} / \mathrm{R}$ injury as to those observed in vivo, we next investigated the role of S1PR2 in MMP activation in brain endothelial cells. Activation of human brain endothelial cells with TNF- $\alpha\left(5 \mathrm{ng} \mathrm{ml}^{-1}\right)$ for $24 \mathrm{~h}$ resulted in increased MMP-9 activity in the conditioned media compared with vehicletreated cells (5.8 \pm 1.2 -fold induction, Fig. 6e). In addition, TNF- $\alpha$ treatment induced the cleavage of pro-MMP-2 $(72 \mathrm{kDa})$ into the 67 and $64 \mathrm{kDa}$ cleaved active forms $(2.3 \pm 0.3$-fold versus control). Blockade of S1PR2 signalling by JTE013 $(1 \mu \mathrm{M})^{20}$ significantly decreased MMP-9 activity in the conditioned media, while it did not significantly change the levels of the cleaved active MMP-2 forms (Fig. 6e). Consistent with the MMP activity data in endothelial conditioned media, we found that MMP-9 mRNA levels in brain endothelial cells were low under basal conditions and upregulated by TNF- $\alpha$ (Supplementary Fig. 5a), while MMP-2 mRNA levels were constitutively high and not affected by TNF- $\alpha$ (Supplementary Fig. 5b). Also, JTE013 partially inhibited the induction of MMP-9 mRNA by TNF- $\alpha$, while it did not affect MMP-2 mRNA levels. These data are consistent with the critical role of S1PR2 in endothelial activation ${ }^{21}$. Using a gain-of-function approach, we found that upregulation of S1PR2 levels by adenoviral transduction in hBMVEC resulted in increased levels of MMP-9 mRNA and MMP-9 activity (Supplementary Fig. $5 \mathrm{c}-\mathrm{e}$ ) compared with control adenovirus-transduced cells ( $\beta$-galactosidase). These data indicate that S1PR2 upregulation in brain endothelial cells is sufficient for the induction of MMP-9 mRNA and MMP-9 activity in endothelial cell-conditioned media. Altogether our in vitro data indicate that S1PR2 plays a critical role in the induction of brain endothelial cell permeability after I/R injury and activation of MMP-9.

S1PR2 is dispensable for neural and glial responses in vitro. To assess the role of S1PR2 in the regulation of neuronal and glial responses to ischaemic and inflammatory injury, we isolated and cultured mouse cortical primary neurons from embryos (E 17.5) and mixed glial cells from pups (post natal day 2). In vitro I/R injury ( $4 \mathrm{~h}$ of OGD followed by in vitro reperfusion) did not significantly change the levels of S1PR2 mRNA in mouse cortical primary neurons (Fig. 7a). In addition, blockade of S1PR2 
a

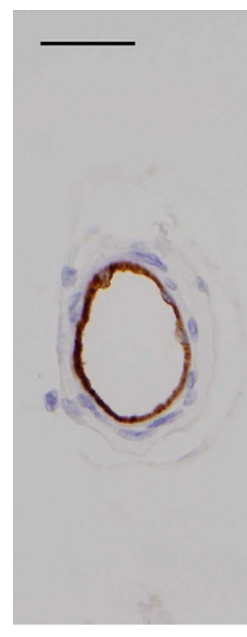

C

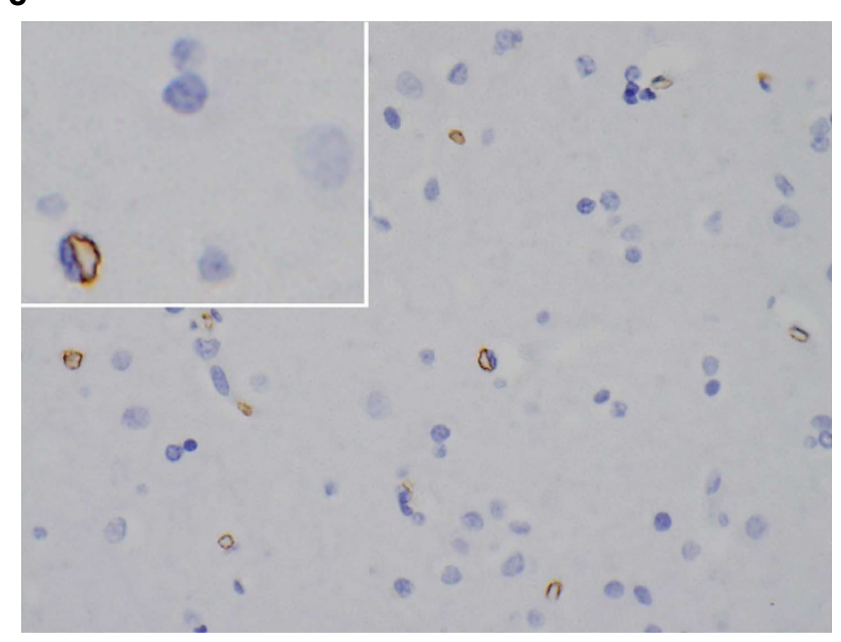

b

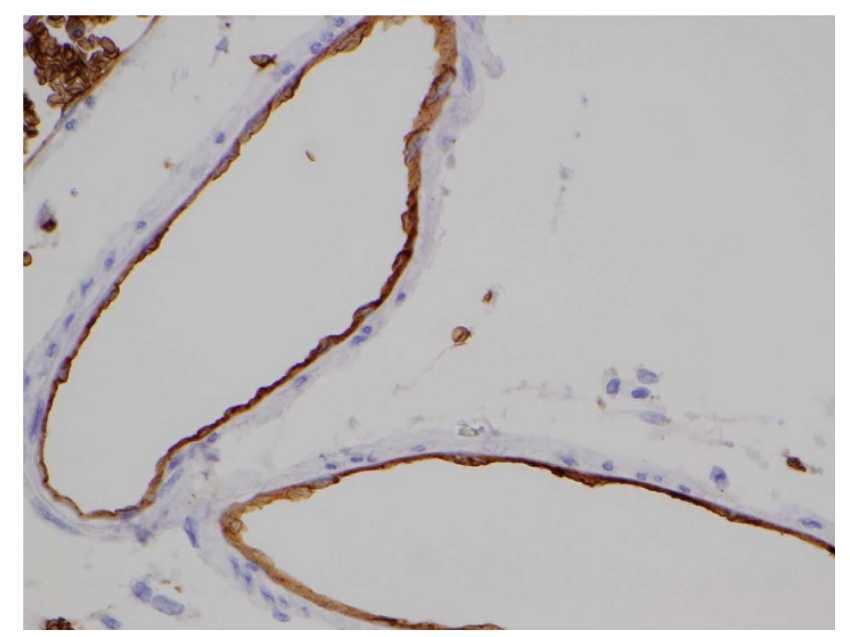

d

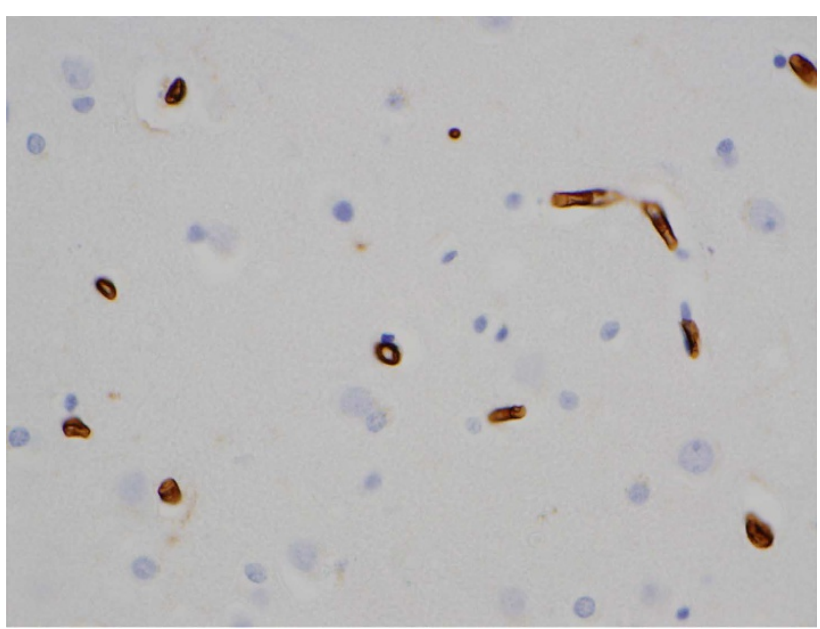

e

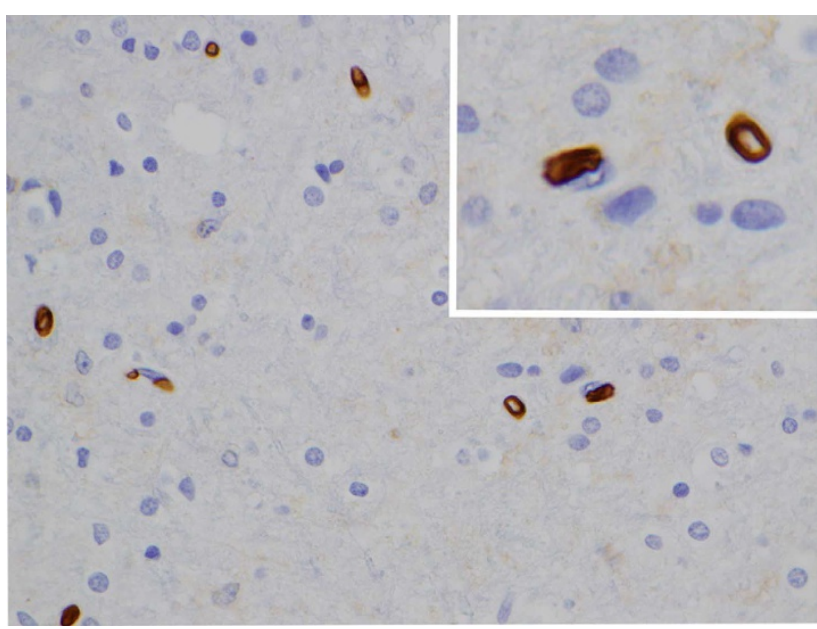

Figure 8 | Immunohistochemical analysis of S1PR2 in human brain. S1PR2 was detected in endothelial cells of pial vessels and microvessels in autopsy specimens. Representative images are shown. (a-e) Cases 1-5. Pictures were taken at $\times 60$ magnification. Scale bar, $30 \mu \mathrm{m}$.

signalling by JTE013 did not inhibit neuronal cell death induced by in vitro I/R injury, assessed by lactate dehydrogenase activity (LDH) activity in neuronal supernatants (Fig. 7b), while $\alpha$-phenyl-tert-butyl nitrone $\alpha$-(PBN), a neuroprotective spin trap agent that reacts with and trap free radicals ${ }^{35}$, did inhibit. Similarly, neuronal cell death induced by $\mathrm{H}_{2} \mathrm{O}_{2}$ was not affected by JTE013 but it was inhibited by $\alpha$-PBN (Fig. 7c). In mouse primary mixed glial cells, activation with TNF- $\alpha$ did not significantly increase the levels of S1PR2 mRNA (Fig. 7d), while it significantly induced the expression of other pro-inflammatory molecules, such as 
interleukin-1 $\beta$ (Fig. 7e). In addition, we found that JTE013 did not affect the inflammatory responses of mouse primary mixed glial cells. As shown in Fig. 7f, the induction of MMP-9 activity by TNF- $\alpha$ in glial cell supernatants was not inhibited by the S1PR2 antagonist, JTE013. These data indicate that S1PR2 is not critical for the regulation of neuronal and glial responses to ischaemic and inflammatory injury in vitro. Altogether our in vitro data with brain endothelial cells, neurons and glial cells indicate that S1PR2 plays a critical role in the responses of brain endothelial cells to I/R injury.

Expression of S1PR2 in human brain samples. Given our in vivo and in vitro findings, to assess their potential pathophysiological relevance in humans we performed immunohistochemical analysis of S1PR2 in human brain autopsy samples. The specificity of the S1PR2 antibody was determined as we have previously described $^{36}$ : S1PR2 immunohistochemistry showed membranous and cytoplasmic staining only in S1PR2-transfected 293T cells and not in S1PR1-transfected or pcDNA3.1-transfected cells (Supplementary Fig. 6). Immunohistochemical analysis of human brain samples revealed S1PR2 positivity in the cerebrovascular endothelium (Fig. 8, representative pictures). We found heterogeneous S1PR2 expression in the endothelium of pial vessels and microvessels throughout the brain in the five brain samples analysed. The limited clinical data available for these patients (summarized in Supplementary Table 1) revealed various hypoxic/ischaemic states including stroke, respiratory failure or sickle cell vaso-occlusive crisis. These data indicate that S1PR2 can be detected in the human cerebrovascular endothelium.

\section{Discussion}

In this study, we have identified the critical role of S1PR2 in the disruption of neurovascular integrity after I/R injury. We found that genetic deletion or pharmacological inhibition of S1PR2 promoted cerebrovascular integrity in a mouse model of transient cerebral ischaemia (tMCAO), resulting in decreased neuronal death and improved neurological scores. Inhibition of S1PR2 potently blocked the development of cerebral oedema and spontaneous haemorrhagic transformation in experimental stroke. We also found that S1PR2 signal is detected in cerebral microvessels in mice in the early stages after $I / R$ injury. In addition, our in vivo and in vitro data provide mechanistic insights into the regulation of cerebrovascular permeability by S1PR2. In vivo, we found that genetic deletion or pharmacological inhibition of S1PR2 results in decreased MMP-9 activity in whole brain lysates and gelatinase (MMP-2/9) activity in cerebral microvessels, which is consistent with the decreased cerebrovascular permeability and haemorrhagic transformation observed in S1pr2 ${ }^{-1-}$ mice or wild-type mice treated with JTE013 compared with vehicle-treated wild-type mice. Our in vitro studies indicate that S1PR2 plays a critical role in the responses of brain endothelial cells to I/R injury (that is, permeability and MMP-9 activation) but not neurons or glial cells. In experimental stroke, MMP have been shown to play a critical role in the blood-brain barrier disruption and haemorrhagic transformation ${ }^{10,37,38}$. More specifically, MMP-9 levels and activity are increased early during cerebral ischaemia ${ }^{38}$ and mice lacking MMP-9 (ref. 8), but not MMP-2 (ref. 39), are protected in stroke models. Interestingly, in stroke patients, increased plasma levels of MMP-9, but not other MMP, have been associated with increased risk of developing haemorrhagic transformation ${ }^{40,41}$. Although endothelial cells, astrocytes, neurons and immune cells can upregulate MMP-9, several studies point to the cerebrovascular endothelium as an important source of MMP-9 activity in the early stages of ischaemia contributing to the early disruption of the blood-brain barrier ${ }^{8,31,42}$. Altogether, our in vivo and in vitro data point to the activation of MMP-9 in the endothelium as a mechanism of induction of cerebrovascular permeability by S1PR2 after I/R injury.

Our findings are of potential translational relevance since the use and effectiveness of current stroke reperfusion therapies are limited by subsequent development of cerebrovascular complications of reperfusion injury (that is, vasogenic oedema and haemorrhagic transformation). Increased cerebrovascular permeability plays a critical role in the pathophysiology of stroke and I/ $\mathrm{R}$ injury by allowing the entrance of neurotoxic plasma components and blood cells into the brain parenchyma, which compromises synaptic and neuronal function and by increasing intracerebral pressure, with the risk of brain herniation and/or compression of cerebral vessels, further compromising blood flow to the brain. In animal models of cerebral ischaemia, blood-brain barrier disruption occurs early and continues after reperfu$\operatorname{sion}^{25,26}$. In stroke patients, early blood-brain barrier disruption is associated with haemorrhagic transformation and poor clinical outcomes ${ }^{29}$. Haemorrhagic transformation is a serious complication that limits the effectiveness and use of current stroke reperfusion therapies (thrombolytic and mechanical recanalization ${ }^{43}$, but it may also spontaneously develop as part of the natural evolution of ischaemic brain injury ${ }^{44}$. In this study, we found that S1PR2 inhibition potently blocked the development of spontaneous haemorrhagic transformation in experimental stroke, which was observed after a more severe ischaemic injury. In addition, our findings that S1pr2-deficient mice or wild-type mice, treated with the S1PR2 antagonist JTE013, exhibited a dramatic decrease in cerebral oedema also raise the intriguing possibility that S1PR2 targeting could be potentially beneficial to prevent the development of malignant MCA syndrome, in which rapid brain oedema following a large MCA infarct leads to herniation and high mortality rates $(80 \%)^{45}$, particularly in younger stroke patients ${ }^{46,47}$. Current therapeutic options to control cerebral oedema in stroke aim at decreasing intracerebral pressure by osmotherapy ${ }^{48}$, hyperventilation, administration of diuretics, induction of coma $^{49}$ or hypothermia, among others ${ }^{50}$. Although most of these conservative treatments have failed to improve mortality and disability ${ }^{49,50}$, they are still being used due to the lack of alternative therapies. Only surgical decompression has been shown to be effective but only in younger patients due to the risk associated with the surgery ${ }^{51}$. In our study, we found that S1PR2 expression is detected in brain microvessels after stroke, which together with our in vitro data with brain endothelial cells, neurons and glial cells, indicate that S1PR2 plays a critical role in the disruption of cerebrovascular integrity. We also found that the S1PR2 antagonist, JTE013, is protective when given within $4.5 \mathrm{~h}$ of stroke onset (therapeutic time window). These findings have a high translational relevance and could potentially develop into novel therapies, especially with the increasing clinical use of thrombolytic or mechanical reperfusion therapies in stroke patients. In contrast to the efforts made in the development of neuroprotective drugs, very few studies have focused on the development of agents that promote cerebrovascular integrity. Our findings suggest that administration of vasoprotective agents (for example, a S1PR2 antagonist) at the time of reperfusion could be a novel strategy to diminish cerebrovascular complications of reperfusion injury, ultimately resulting in less neurovascular injury and improved stroke outcomes ${ }^{52-54}$ (Fig. 9).

The pathophysiological relevance of our in vivo findings in mice is strengthened by the fact that we detected the expression of S1PR2 in the cerebrovascular endothelium in human brain samples. More specifically, we have detected S1PR2 expression in the cerebrovascular endothelium of pial vessels and microvessels 


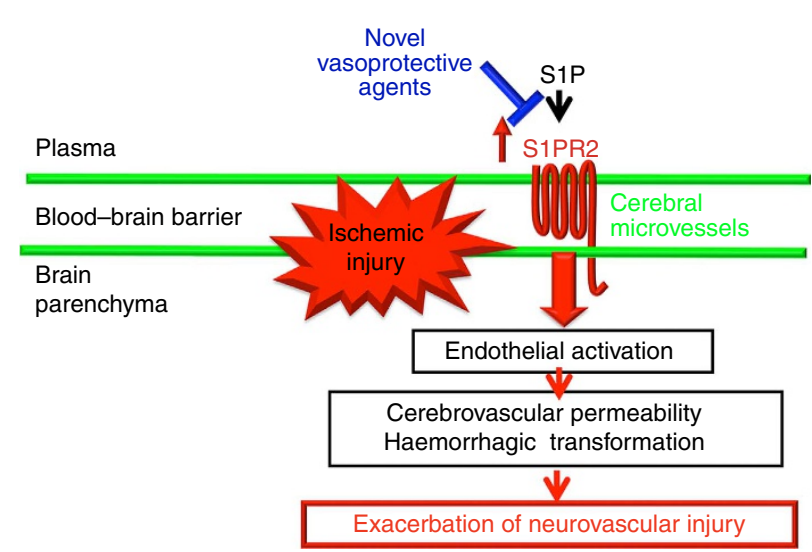

Figure 9 | Critical role of S1PR2 in the disruption of cerebrovascular integrity in stroke. Diagram summarizing our findings and the potential of S1PR2 antagonists as novel neurovascular protective therapies in stroke.

in human tissue. Our human study is limited by the fact that only autopsy material was available and also by the low number of cases. A series of a larger number of cases, and ideally biopsy specimens or autopsy material collected within few hours after death, would be required to more definitively characterize the regulation of S1PR2 expression in the cerebrovascular endothelium after ischaemic/hypoxic/inflammatory injury in the human brain. Nevertheless, we can conclude that S1PR2 is detected in the human cerebrovascular endothelium, which is consistent with a recent report by Cruz-Orengo et al. ${ }^{55}$ in which they found that S1PR2 is upregulated in cerebellar and spinal cord microvessels in multiple sclerosis lesions. Altogether our data suggest that S1PR2 is a novel and attractive target, which deserves further study in terms of the potential for pharmacological blockade to promote cerebrovascular integrity in humans.

S1PR are becoming attractive candidates for drug discovery. The most well-characterized S1PR, S1PR subtype 1, was originally cloned from human endothelial cells ${ }^{56}$, and plays a critical role in vascular integrity 18,19 and lymphocyte trafficking and egress from lymphoid organs ${ }^{22,57,58}$. In fact, the recently approved drug by FDA for the treatment of multiple sclerosis, FTY720, a potent immunosuppressor, is an agonist of S1PR1, 3, 4 and 5. In recent studies, FTY720 has been shown to confer neuroprotection in animal models of stroke ${ }^{59,60}$ via its immunosuppressive effects ${ }^{60}$ but not by direct neuroprotection ${ }^{59,60}$. However, FTY720 treatment is associated with increased risk of infection in multiple sclerosis patients ${ }^{61}$. Increasing clinical and preclinical evidence indicates that stroke-induced immunosuppression is the cause of increased vulnerability of stroke patients to infection ${ }^{62,63}$, a leading cause of prolonged intensive care unit stays and increased mortality in stroke patients. Therefore, further research is still needed before considering immunomodulation (for example, FTY720) as a therapeutic alternative for human stroke. In contrast to these previous reports ${ }^{59,60}$, our study is focused on a different S1P receptor, S1PR2, which is not targeted by FTY720. Our recent work has identified endothelial S1PR2 as a robust mediator of vascular permeability and acute vascular injury in several organs in sepsis models ${ }^{21}$. In addition, we found no differences in immune cell trafficking between wild-type and S1pr2 null mice ${ }^{21}$. Therefore, targeting of this novel pathway would have a potential advantage compared with targeting S1PR1 since it could lead to neurovascular protection without compromising immune function, which could be especially beneficial for stroke patients.

Recent studies from our lab indicate that S1PR2 plays a role in endothelial activation (permeability and inflammation) during acute inflammatory vascular injury ${ }^{20,21}$. On activation with pro-inflammatory stimuli, SPHK-1 activity, the rate-limiting enzyme in S1P generation, is increased in endothelial cells ${ }^{64}$ and S1P is released. S1PR2 expression is also induced in endothelial cells during inflammation, while the levels of the other receptors do not change significantly ${ }^{21}$. Our in vitro data is consistent with a model in which the binding of endothelialderived or serum-derived S1P to S1PR2 is blocked by JTE013, resulting in inhibition of brain endothelial cell activation (for example, permeability and MMP-9 activity) after in vitro I/R injury or inflammatory challenge. The induction of MMP-9 by S1PR2 likely involves the activation of the Rho-ROCK-NFKB and the stress-activated protein kinase pathways ${ }^{12}$, two propermeability and pro-inflammatory pathways that are activated by S1PR2 (ref. 21). Although S1PR2 is emerging as an important mediator of endothelial dysfunction in septic/inflammatory and ischaemic vascular injury ${ }^{20,21}$, it has been shown to play a protective role in anaphylactic shock via inhibition of endothelial nitric oxide synthase ${ }^{65,66}$. Therefore, the effect of S1PR2 activation in terms of exacerbating or ameliorating disease appears to depend on the underlying pathophysiological setting. A better understanding of the molecular mechanisms involved in the regulation of S1PR2 expression in different vascular beds and cell lineages, as well as its downstream molecular mediators is required to determine appropriate targeting strategies of the S1PR2 pathway in various pathophysiological settings.

In conclusion, our data indicate that S1PR2 plays a critical role in the disruption of neurovascular integrity after I/R injury and MMP-9 activation in brain endothelial cells. These findings reveal the novel role of S1PR2 in the pathophysiology of cerebrovascular complications of $\mathrm{I} / \mathrm{R}$ injury in experimental stroke. Altogether our in vitro and in vivo data in mice and humans point to S1PR2 as an attractive therapeutic target for cerebrovascular protection in stroke and other instances of hypoxic or inflammatory injury in cerebral blood vessels.

\section{Methods}

Mice. Mice with targeted disruption of the S1pr2 gene ${ }^{23}$ were maintained on a mixed C57BL/6;129Sv genetic background (five times backcrossed to C57BL/6) Experiments on knockout mice were performed with appropriate wild-type littermate controls. All animal studies were approved by the Beth Israel Deaconess Medical Center Institutional Animal Care and Use Committee.

tMCAO and treatments. MCAO was performed using wild-type and knockout male mice $(25-30 \mathrm{~g})$ as previously described ${ }^{24,67}$. In brief, surgery was performed using a dissecting surgical microscope. Temperature was maintained at $36.5-37^{\circ} \mathrm{C}$, controlled by a thermostatic blanket (TC-1000 Temperature Controller, CWE, USA) throughout the procedure. Mice were anesthetized with isoflurane, delivered by facemask in $\mathrm{O}_{2}$-enriched air. Blood flow in the MCA territory was monitored throughout the procedure using by a laser Doppler probe (PeriFlux system 5000, Ardmore, PA, USA), placed on the skull directly over the territory of the left MCA perfusion area. A midline incision was made in the neck (1.5- $\mathrm{cm}$ long), the common carotid and external carotid arteries were dissected from the adjacent tissue. After occlusion of the common carotid by a microclip, the left external carotid was ligated, coagulated and cut. Nylon monofilament (6-0) with a round tip $(\sim 0.22 \mathrm{~mm}$ ) was introduced gently up to $\sim 8.5-9 \mathrm{~mm}$ towards the origin of the MCA. The successful occlusion was verified by laser Doppler flowmetry (75-90\% blood flow decrease of the baseline). After the successful occlusion, the nylon monofilament was secured in place by ligation. About 90 or $180 \mathrm{~min}$ after occlusion, the suture was withdrawn to allow reperfusion. Animals were excluded from the experimental group only if the CBF did not decrease by $75-90 \%$ during occlusion or it did not recover up to $70 \%$ of baseline within 10 min after the start of reperfusion (a total of eight animals were excluded from the study). About $10 \mathrm{~min}$ after reperfusion, animals were randomly assigned to the treatment groups: JTE013 $\left(10 \text { or } 30 \mathrm{mg} \mathrm{kg}^{-1} \text { by gavage }\right)^{21}$ or vehicle ( $2 \% 2$-hydroxypropyl $\beta$-cyclodextrin in saline). To determine the therapeutic time window of JTE013 administration, vehicle or $30 \mathrm{mg} \mathrm{kg}^{-1}$ JTE013 were administered $7.5,4.5$ or $1.7 \mathrm{~h}$ (10 min after reperfusion) after the onset of ischaemia. For the 3-h time point assays (for example, determination of cerebrovascular permeability), JTE013 or vehicle was administered just before occlusion. After the surgery, all animals were maintained in a small animal heated recovery chamber (IMS Vetcare Chamber Recovery Unit, Harvard Apparatus, Holliston, MA). 
TTC staining and determination of infarct and oedema ratios. Staining with 2,3,5-triphenyltetrazolium chloride (TTC) was carried out by an independent investigator to identify viable and nonviable brain tissue. Because TTC is metabolized by intact mitochondria, the red areas indicate the living tissue. Brains were harvested, sectioned into 1-mm-thick slices and incubated in a $1 \%$ solution of TTC in phosphate-buffered saline at $37^{\circ} \mathrm{C}$ for $5-10 \mathrm{~min}$. Scanned images were used to calculate infarct and oedema ratios using image analysis software (Image J, the National Institutes of Health, Bethesda, MD). Infarct ratios were obtained after normalization by the contralateral (non-ischaemic) hemisphere and corrected for oedema as previously described ${ }^{67}$. Briefly, the infarct ratio $(I)$, corrected for oedema, was calculated by using the following equation: $I=(X-S) / Z$, where $X$ is the area of infarct $\left(\mathrm{mm}^{2}\right), S$ is brain swelling $\left(\mathrm{mm}^{2}\right), S=Y-Z, Y$ is the area of the infarcted (ipsilateral) hemisphere slice $\left(\mathrm{mm}^{2}\right)$ and $Z$ is the area of the non-infarcted (contralateral) hemisphere slices $\left(\mathrm{mm}^{2}\right)$. The oedema ratio was calculated with the following formula: $E=(Y-Z) / Z$.

Neurological score evaluation. Neurologic examinations were carried out by an independent investigator $24 \mathrm{~h}$ after tMCAO. Neurological scores were calculated as followed: 0: no deficit, 1: forelimb weakness and torso turning to the ipsilateral side when held by tail, 2: circling to affected side when held by tail on the bench, 3: spontaneous circling to the affected side or unable to bear weight on the affected side and 4: no spontaneous locomotor activity or barrel rolling ${ }^{67}$.

In situ cell death detection. DNA strand breakage during apoptosis after ischaemic brain damage was assessed by a TUNEL method using the In Situ Cell Death Detection Kit, TMR red (Roche Applied Science, Manheim, Germany), according to the manufacturer's instructions. Briefly, brain sections $(30 \mu \mathrm{m})$ were permeabilized ( $0.1 \%$ Triton X-100, $0.1 \%$ sodium citrate solution) for $5 \mathrm{~min}$ on ice and then incubated with TUNEL reaction mixture (1:10, enzyme solution/label solution) for $1 \mathrm{~h}$ at $37^{\circ} \mathrm{C}$. DAPI (4',6-diamidino-2-phenylindole) staining was used as a counter staining. Images were captured with the Axio Imager A1 microscope, using AxioCam MRc camera and the AxioVision 4.8 programme (Carl Zeiss Inc.) (original magnification, $\times 40$ ). For quantification, the number of TUNEL-positive cells/field from three different fields in the damaged area per mouse (bregma +1.2 to $+0.8 \mathrm{~mm}$, rostrally) were counted and the average values were plotted.

EBD extravasation assay. To assess cerebrovascular permeability, $1 \mathrm{~h}$ after reperfusion, a $2 \% \mathrm{EBD}$ solution $\left(4 \mathrm{~mL} \mathrm{~kg}^{-1}\right)$ was injected into the lateral tail vein. EBD binds to plasma albumin and it can be quantified in the extravascular compartment if there is increased vascular permeability. After $2 \mathrm{~h}$, animals were anesthetized and perfused through the left ventricle with ice-cold PBS to remove intravascular EBD. Brains were harvested, sliced and scanned. Then, the hemispheres were separated and mechanically homogenized in $50 \%$ trichloroacetic solution. After centrifugation (20,000 g for $20 \mathrm{~min})$, supernatants were diluted 1:3 with ethanol. The amount of extravascular EBD was calculated by measuring the fluorescence (620 excitation, 680 emission). Ipsilateral/contraleral ratios were calculated and plotted.

Quantification of extravascular haemoglobin. To assess haemorrhagic transformation, $24 \mathrm{~h}$ after a 3 -h occlusion period, mice were anesthetized and perfused through the left ventricle with a $5 \mathrm{mM}$ EDTA-saline solution. Brains were harvested, sliced and scanned. Then, the ipsilateral and contralateral hemispheres were separated and homogenized in PBS. After sonication, the homogenates were centrifuged at 13,000 r.p.m. for $30 \mathrm{~min}$ at $4^{\circ} \mathrm{C}$ and extravascular haemoglobin was quantified using the QuantiChrom TM Hemoglobin Assay Kit (BioAssay Systems, Hayward, CA) following manufacturer's instructions. The amount of haemoglobin in brain homogenates was calculated using a standard curve. Ipsilateral/contraleral ratios were calculated and plotted.

Determination of gelatinase activity in brain lysates. Three hours after reperfusion mice were perfused through the left ventricle with ice-cold PBS and brains were removed and frozen in liquid nitrogen. Brain tissue was homogenized in lysis buffer $\left(50 \mathrm{mmoll}^{-1}\right.$ Tris-HCL pH $7.6,150 \mathrm{mmoll}^{-1} \mathrm{NaCl}, 5 \mathrm{mmoll}^{-1}$ $\mathrm{CaCl} 2,0.05 \%$ Brij-35, $0.02 \% \mathrm{NaN} 3$ and $1 \%$ Triton X-100) and centrifuged at 13,000 r.p.m. for $20 \mathrm{~min}^{10}$. About $0.8 \mathrm{mg}$ protein in $500 \mu \mathrm{l}$ lysis buffer was incubated with $40 \mu \mathrm{l}$ (bead volume) of gelatin-Sepharose 4B beads (GE Healthcare, Piscataway, NJ, USA) for $1 \mathrm{~h}$ at $4{ }^{\circ} \mathrm{C}$ with gentle rotation to pull down MMP from brain homogenates. The beads were washed three times with lysis buffer and were resuspended in $30 \mu \mathrm{l}$ of elution buffer (10\% dimethylsulfoxide in lysis buffer) for $30 \mathrm{~min}$. The supernatants were collected and then each sample was mixed with equal amounts of SDS sample buffer (Invitrogen Life Technologies, Carlsbad, CA, USA). The samples were loaded on $10 \%$ SDS-polyacrylamide electrophoresis gels with $0.1 \%$ porcine skin gelatin as a substrate (Invitrogen Life Technologies). After electrophoresis, the gels were incubated with renaturing buffer $(2.5 \%$ Triton X-100 in $\mathrm{H}_{2} \mathrm{O}$ ) for $30 \mathrm{~min}$ to remove the SDS and then incubated for $24 \mathrm{~h}$ (for MMP-2) or $48 \mathrm{~h}$ (for MMP-9) at $37^{\circ} \mathrm{C}$ with developing buffer (Invitrogen Life Technologies). After incubation, gels were stained for $60 \mathrm{~min}$ in Coomassie blue R-250 $(0.25 \%$ Coomassie brilliant blue R-250, 50\% methanol, 10\% acetic acid) and de-stained in
$40 \%$ methanol and $10 \%$ acetic acid until clear bands of gelatinolysis appeared on a blue background. Human MMP-2 and MMP-9 were used as a gelatin standard (Chemicon International Inc. Temecula, CA, USA). The images were scanned and quantification was performed using Image J programme. Full gels are shown in Supplementary Fig. 7.

In situ zymography in ischaemic brain tissue. Mice were killed $3 \mathrm{~h}$ after reperfusion and perfused through the left ventricle with ice-cold PBS. Brains were removed, quickly frozen and kept at $-80^{\circ} \mathrm{C}$. To assess gelatinase activity in situ, fresh sham and ischaemic brain slices $(14 \mu \mathrm{m})$ were incubated with a reaction solution including DQ-gelatin conjugated with fluorescein (Life technologies, Carlsbad, CA) for $6 \mathrm{~h}$ at $37^{\circ} \mathrm{C}$. Sections were then washed three times with PBS and subsequently incubated with DAPI for nuclear staining. Images were captured with the Axio Imager A1 microscope, using AxioCam MRc camera and the AxioVision 4.8 programme (original magnification, $\times 40$ ).

Immunostaining for S1PR2 detection. Mice were anesthetized with Avertin and were perfused with cold PBS and subsequently with $4 \%$ PFA in PBS solution. The brains were removed, post-fixed with $4 \%$ PFA for $24 \mathrm{~h}$ and transferred to $30 \%$ sucrose solution. Frozen brains were cut with a thickness of $30 \mu \mathrm{m}$ in a cryostat. Slices were preserved in 30\%PEG $30 \%$ sucrose PBS. The brain slices were washed three times with Tris-buffered saline (TBS) and were then blocked with TBSblocking solution ( $1 \%$ bovine serum albumin, $0.2 \%$ skim milk and $0.3 \%$ Triton $\mathrm{X}-100$ in TBS) for $1 \mathrm{~h}$ and incubated with the following primary antibodies in TBSblocking solution overnight on a shaker at $4^{\circ} \mathrm{C}$. Rabbit anti-S1P2R antibody $(1: 100$, Proteintech Group Inc., Chicago, IL) and rat anti-CD31 antibody (1:100, BD Biosciences, Pharmingen, San Jose, CA) were used as primary antibodies. Sections were washed three times with TBS and then they were incubated with donkey antirabbit IgG Alexa-594 and goat anti-rat IgG Alexa-488 (1:250, Life Technologies, Molecular Probes, Grand Island, NY). Brain slices were stained with DAPI for $7 \mathrm{~min}$ and were mounted onto slides. Samples were observed on a Zeiss LSM 510 Meta Confocal microscope (Carl Zeiss Inc.). Images were captured using Zen LE software (Carl Zeiss). The specificity of the S1PR2 antibody was confirmed by lack of signal in the S1pr2 ${ }^{-1-}$ brain sections.

Brain endothelial cell culture and in vitro I/R studies. The mouse brain endothelial cell line bEnd.3 (passages 25-35; ATCC) ${ }^{33}$ was cultured in DMEM supplemented with 10\% FBS. Passages 25-35 were used. hMVBEC (Cell Systems Corporation, Kirkland, WA) were cultured as recommended by the manufacturer. Passages 5-9 were used.

To mimic in vitro the metabolic and inflammatory stress of I/R injury, OGD and TNF- $\alpha$ activation studies were conducted.

Brain endothelial cells were exposed to hypoxia $\left(1 \% \mathrm{O}_{2}\right)$ /aglycemia for $6 \mathrm{~h}$ in a hypoxia polymer glove box (Coy Lab Products Inc., MI). Then, the media was aspirated and replaced with complete growth media (which contains glucose) and cells were placed in a normoxic atmosphere. For the permeability studies, the media contained serum and changes in endothelial resistance after reperfusion were measured using the ECIS ${ }^{34}$. For the TNF- $\alpha$ activation studies, cells were stimulated with $5 \mathrm{ng} \mathrm{ml}^{-1}$ TNF- $\alpha$ in serum-free media containing growth factors (Cell Systems Corporation).

Assessment of brain endothelial cell permeability. bEnd 3.3 cells were grown to confluence in disposable electrode arrays containing gold film electrodes (Applied BioPhysics), previously coated with human fibronectin and collagen IV $\left(50 \mu \mathrm{g} \mathrm{ml}^{-1}\right)$ and exposed to hypoxia $\left(1 \% \mathrm{O}_{2}\right) /$ aglycemia for $6 \mathrm{~h}$ in a hypoxia polymer glove box. Then, the media was aspirated and replaced with complete growth media (which contains glucose and 10\% foetal bovine serum), cells were placed in a normoxic atmosphere and changes in endothelial resistance on reperfusion were measured using the ECIS ${ }^{34}$

MMP activity in brain endothelial cell supernatants. For in vitro detection of MMP-2 and MMP-9 activity, conditioned media from hBMVEC subjected to TNF- $\alpha$ treatment were used. hBMVEC were grown on 12-well plates. Cells were treated with TNF- $\alpha\left(5 \mathrm{ng} \mathrm{ml}^{-1}\right)$ in serum-free complete growth media (Cell Systems Corporation) for $24 \mathrm{~h}$. Conditioned media were collected, spun down, $10 \mu \mathrm{l}$ of supernatants were mixed with equal amounts of SDS sample buffer and gelatin zymography was conducted as described above.

Primary cortical neuron isolation and in vitro I/R studies. Mouse (C57BL6) E16.5 embryos were used for primary cortical neuron isolation as previously described ${ }^{68}$. Briefly, the cortices were collected and incubated with $0.25 \%$ trypsin for $15 \mathrm{~min}$ at $37^{\circ} \mathrm{C}$ for tissue digestion. Foetal bovine serum was added to stop the trypsin activity. After centrifugation, the supernatant was discarded and DMEM complete media containing $10 \%$ FBS and antibiotics were added to the cells. The cell suspension was passed through $70 \mu \mathrm{m}$ cell strainer (BD Biosciences, Falcon) and was plated on poly-L-lysine (Sigma Aldrich, St Louis, MO) coated dishes with DMEM complete media. The next day, the medium was changed to Neurobasal 
media containing B27 supplement, antibiotics and GlutaMAX (Life Technologies). At 7 days in vitro, the cells ( $>95 \%$ NeuN positive) were used for the in vitro I/R injury assays. Neurons were subjected to hypoxia $\left(1 \% \mathrm{O}_{2}\right) /$ aglycemia for $4 \mathrm{~h}$ in a hypoxia polymer glove box. Then, the media was aspirated and replaced with complete growth media (which contains glucose) in the presence or absence of vehicle, $\alpha$-PBN or JTE013 and cells were placed in a normoxic atmosphere. Cells were harvested for RNA isolation, quantitative reverse transcription-PCR analysis (qRT-PCR), 6 or $24 \mathrm{~h}$ after in vitro reperfusion to determine S1PR2 mRNA levels. LDH activity was assessed $24 \mathrm{~h}$ after reperfusion. To mimic the oxidative stress characteristic of I/R injury, neurons were treated with $30 \mu \mathrm{M} \mathrm{H}_{2} \mathrm{O}_{2}$ in the presence or absence of vehicle, $\alpha$-PBN or JTE013 for $24 \mathrm{~h}$ and LDH activity was assessed in neuronal supernatants.

LDH assay. Neuronal cytotoxicity was assessed by measuring LDH activity in the media, $24 \mathrm{~h}$ after in vitro I/R injury or $\mathrm{H}_{2} \mathrm{O}_{2}$ treatment, using the Cytotoxicity Detection Kit (Roche Applied Science), as instructed by the manufacturer. Data were plotted as fold change compared with non-treated control cells. $\alpha$-PBN was used as a positive control for oxidative stress-induced cell death blocker ${ }^{35}$.

Mixed glial cell culture and activation. Mixed glial cell culture was prepared using the mouse brains from postnatal day 2 mouse. Briefly, cerebral cortices were dissected, trypsinized with of $0.25 \%$ trypsin-EDTA in Hank's Balance Salt Solution (HBSS) and were incubated with trypsin (Thermo scientific) and DNase (Worthington) for $15 \mathrm{~min}$ at $37^{\circ} \mathrm{C}$. Foetal bovine serum was added to the cell suspension to stop trypsin digestion. Cell suspension was centrifuged and the pellet was resuspended with DMEM containing $20 \%$ FBS and antibiotics. Cell suspension was filtered with a $100-\mu \mathrm{m}$ cell strainer (BD Falcon) into another $50 \mathrm{ml}$ conical tube. Cells were plated onto six-well plates, which were pre-coated with poly-Dlysine. Three days after plating, the media was changed to DMEM containing $10 \%$ FBS and antibiotics. Cells were maintained in DMEM containing 10\% FBS and antibiotics at $37^{\circ} \mathrm{C}$ with $5 \% \mathrm{CO} 2$, with a medium change every 3 days. At day 10 after plating, mixed glial cells were treated with $5 \mathrm{ng} \mathrm{ml}^{-1} \mathrm{TNF}-\alpha$ to mimic the pro-inflammatory environment of $\mathrm{I} / \mathrm{R}$ injury. About $24 \mathrm{~h}$ later, MMP activity was assessed in the supernatants as described below and glial cells were harvested for RNA isolation and qRT-PCR analysis to determine S1PR2 mRNA levels.

S1PR2 Immunohistochemistry. Immunohistochemistry for S1PR2 was performed on an automated stainer (Leica Bond III, Leica Biosystems, Buffalo Grove, IL) using an anti-human S1PR2 rabbit polyclonal antibody (catalogue \# HPA014307, Sigma Aldrich) at a final dilution of $2 \mu \mathrm{g} \mathrm{ml}^{-1}$. Cell pellets and $5-\mu \mathrm{m}$ formalin-fixed paraffin-embedded tissue sections were deparaffinized and processed using heatinduced epitope retrieval with an EDTA-based buffer (Leica \# AR9640) for 20 min and incubated with primary antibody for $30 \mathrm{~min}$ at room temperature. Post-primary reagent (polymer anti-rabbit poly-horseradish peroxidase-IgG,

$<25 \mu \mathrm{g} \mathrm{ml}^{-1}$, Leica \# DS9800) was incubated for $10 \mathrm{~min}$ at room temperature and staining was visualized with diaminobenzidine for $10 \mathrm{~min}$ (Leica \# DS9800). Pictures were taken with a $\times 60$ objective using Olympus BX41 Camera and CellSens Entry Imaging Software. Cases were retrieved from Brigham and Women's Department of Pathology archives and this work was approved by the Institutional Review Board (Protocol \#2013P001431).

RNA isolation. To prepare mouse organ tissue lysates, mice were perfused with PBS containing $2 \mathrm{mM}$ EDTA, organs were removed and snap frozen in liquid nitrogen. Organs were homogenized and total RNA was prepared using RNeasy Mini Kit (Qiagen, Valencia, CA) with RNase-free DNase treatment (Qiagen) to remove the genomic DNA.

TaqMan qRT-PCR analysis. To generate complementary DNA (cDNA), $100 \mathrm{ng}$ of RNA was reverse transcribed using random primers and SuperScript II RT-polymerase (Invitrogen, Carlsbad, CA). Primers were designed using the Primer Express oligo design programme software (Applied Biosystems, Foster City, CA). All primer sets were subjected to rigorous database searches to identify potential conflicting transcript matches to pseudogenes or homologous domains within related genes. PCR primer sequences for target molecules were previously described $^{21}$ and shown in Supplementary Table 2. Amplicons generated from the primer set were analysed for melting point temperatures using the first derivative primer melting curve software supplied by Applied BioSystems. Real-time quantitative PCR was performed using the SYBR Green I assay on the ABI 7500 Sequence Detection System (Applied Biosystems). PCR reactions for each cDNA sample were performed in duplicate and copy numbers were calculated using standard curves generated from a master template as previously described ${ }^{69}$. Briefly, each cDNA template was purified, precisely quantified and 10 -fold serially diluted to produce a series in which the lowest-concentration dilution has a single copy per microlitre. To generate a standard curve, the threshold cycle (Ct) values for each dilution in the template series are plotted as a function of the logarithm of the known input template numbers and a linear trendline is fitted to the data.
Statistical analyses, randomization and blinding. All values reported are mean \pm s.e.m. $P$ values were calculated with GraphPad Prism software, using (i) one-way ANOVA followed by Newman-Keuls test or (ii) Student's $t$-test when comparing two groups. The criterion for statistical significance was set at $P<0.05$.

All animal experiments used randomization to treatment groups and blinded assessment $^{70}$

\section{References}

1. Lloyd-Jones, D. et al. Heart disease and stroke statistics-2009 update: a report from the American Heart Association Statistics Committee and Stroke Statistics Subcommittee. Circulation 119, 480-486 (2009).

2. Fisher, M. \& Schaebitz, W. An overview of acute stroke therapy: past, present, and future. Arch. Intern. Med. 160, 3196-3206 (2000).

3. Savitz, S. I. \& Fisher, M. Future of neuroprotection for acute stroke: in the aftermath of the SAINT trials. Ann. Neurol. 61, 396-402 (2007).

4. Saver, J. L. Improving reperfusion therapy for acute ischaemic stroke. J. Thromb. Haemost. 9(Suppl 1): 333-343 (2011).

5. Molina, C. A. \& Alvarez-Sabin, J. Recanalization and reperfusion therapies for acute ischaemic stroke. Cerebrovasc. Dis. 27(Suppl 1): 162-167 (2009).

6. Hamann, G. F., Okada, Y. \& Del Zoppo, G. J. Hemorrhagic transformation and microvascular integrity during focal cerebral ischemia/reperfusion. J. Cereb. Blood Flow Metab. 16, 1373-1378 (1996).

7. Del Zoppo, G. J. Inflammation and the neurovascular unit in the setting of focal cerebral ischemia. Neuroscience 158, 972-982 (2009).

8. Asahi, M. et al. Effects of matrix metalloproteinase- 9 gene knock-out on the proteolysis of blood-brain barrier and white matter components after cerebral ischemia. J. Neurosci. 21, 7724-7732 (2001).

9. Guo, S., Stins, M., Ning, M. \& Lo, E. H. Amelioration of inflammation and cytotoxicity by dipyridamole in brain endothelial cells. Cerebrovasc. Dis. 30, 290-296 (2010).

10. Yang, Y., Estrada, E. Y., Thompson, J. F., Liu, W. \& Rosenberg, G. A. Matrix metalloproteinase-mediated disruption of tight junction proteins in cerebral vessels is reversed by synthetic matrix metalloproteinase inhibitor in focal ischemia in rat. J. Cereb. Blood Flow Metab. 27, 697-709 (2007).

11. Murata, Y. et al. Extension of the thrombolytic time window with minocycline in experimental stroke. Stroke 39, 3372-3377 (2008).

12. Candelario-Jalil, E., Yang, Y. \& Rosenberg, G. A. Diverse roles of matrix metalloproteinases and tissue inhibitors of metalloproteinases in neuroinflammation and cerebral ischemia. Neuroscience 158, 983-994 (2009).

13. Pappu, R. et al. Promotion of lymphocyte egress into blood and lymph by distinct sources of sphingosine-1-phosphate. Science 316, 295-298 (2007).

14. Venkataraman, K. et al. Vascular endothelium as a contributor of plasma sphingosine 1-phosphate. Circ. Res. 102, 669-676 (2008).

15. Sanchez, T. \& Hla, T. Structural and functional characteristics of S1P receptors. J. Cell. Biochem. 92, 913-922 (2004).

16. Igarashi, J. \& Michel, T. Sphingosine 1-phosphate and isoform-specific activation of phosphoinositide 3-kinase beta. Evidence for divergence and convergence of receptor-regulated endothelial nitric-oxide synthase signalling pathways. J. Biol. Chem. 276, 36281-36288 (2001).

17. Lee, M. J. et al. Vascular endothelial cell adherens junction assembly and morphogenesis induced by sphingosine-1-phosphate. Cell 99, 301-312 (1999).

18. Liu, Y. et al. Edg-1, the G protein-coupled receptor for sphingosine-1phosphate, is essential for vascular maturation. J. Clin. Invest. 106, 951-961 (2000).

19. Sanchez, T. et al. Phosphorylation and action of the immunomodulator FTY720 inhibits vascular endothelial cell growth factor-induced vascular permeability. J. Biol. Chem. 278, 47281-47290 (2003).

20. Sanchez, T. et al. Induction of vascular permeability by the sphingosine-1phosphate receptor-2 (S1P2R) and its downstream effectors ROCK and PTEN. Arterioscler. Thromb. Vasc. Biol. 27, 1312-1318 (2007).

21. Zhang, G. et al. Critical role of sphingosine-1-phosphate receptor 2 (S1PR2) in acute vascular inflammation. Blood 122, 443-455 (2013).

22. Brinkmann, V. et al. The immune modulator FTY720 targets sphingosine 1-phosphate receptors. J. Biol. Chem. 277, 21453-21457 (2002).

23. Kono, M. et al. The sphingosine-1-phosphate receptors S1P1, S1P2, and S1P3 function coordinately during embryonic angiogenesis. J. Biol. Chem. 279, 29367-29373 (2004)

24. Yang, L., Shah, K., Wang, H., Karamyan, V. T. \& Abbruscato, T. J. Characterization of neuroprotective effects of biphalin, an opioid receptor agonist, in a model of focal brain ischemia. J. Pharmacol. Exp. Ther. 339, 499-508 (2011).

25. Kuroiwa, T., Ting, P., Martinez, H. \& Klatzo, I. The biphasic opening of the blood-brain barrier to proteins following temporary middle cerebral artery occlusion. Acta Neuropathol. 68, 122-129 (1985).

26. Gasche, Y., Copin, J. C., Sugawara, T., Fujimura, M. \& Chan, P. H. Matrix metalloproteinase inhibition prevents oxidative stress-associated blood-brain barrier disruption after transient focal cerebral ischemia. J. Cereb. Blood Flow Metab. 21, 1393-1400 (2001). 
27. Kastrup, A. et al. Early disruption of the blood-brain barrier after thrombolytic therapy predicts haemorrhage in patients with acute stroke. Stroke 39, 2385-2387 (2008).

28. Hjort, N. et al. MRI detection of early blood-brain barrier disruption: parenchymal enhancement predicts focal hemorrhagic transformation after thrombolysis. Stroke 39, 1025-1028 (2008).

29. Latour, L. L., Kang, D. W., Ezzeddine, M. A., Chalela, J. A. \& Warach, S. Early blood-brain barrier disruption in human focal brain ischemia. Ann. Neurol. 56, 468-477 (2004)

30. Khatri, P., Wechsler, L. R. \& Broderick, J. P. Intracranial haemorrhage associated with revascularization therapies. Stroke 38, 431-440 (2007)

31. Romanic, A. M., White, R. F., Arleth, A. J., Ohlstein, E. H. \& Barone, F. C. Matrix metalloproteinase expression increases after cerebral focal ischemia in rats: inhibition of matrix metalloproteinase-9 reduces infarct size. Stroke 29, 1020-1030 (1998).

32. Gasche, Y. et al. Early appearance of activated matrix metalloproteinase-9 after focal cerebral ischemia in mice: a possible role in blood-brain barrier dysfunction. J. Cereb. Blood Flow Metab. 19, 1020-1028 (1999).

33. Montesano, R. et al. Increased proteolytic activity is responsible for the aberrant morphogenetic behavior of endothelial cells expressing the middle $\mathrm{T}$ oncogene. Cell 62, 435-445 (1990).

34. Garcia, J. G. et al. Sphingosine 1-phosphate promotes endothelial cell barrier integrity by Edg-dependent cytoskeletal rearrangement. J. Clin. Invest. 108, 689-701 (2001).

35. Kuroda, S., Katsura, K., Hillered, L., Bates, T. E. \& Siesjo, B. K. Delayed treatment with alpha-phenyl-N-tert-butyl nitrone (PBN) attenuates secondary mitochondrial dysfunction after transient focal cerebral ischemia in the rat. Neurobiol. Dis. 3, 149-157 (1996).

36. Kluk, M. J. et al. Sphingosine-1-phosphate receptor 1 in classical Hodgkin lymphoma: assessment of expression and role in cell migration. Lab. Invest. 93, 462-471 (2013).

37. Sumii, T. \& Lo, E. H. Involvement of matrix metalloproteinase in thrombolysisassociated hemorrhagic transformation after embolic focal ischemia in rats. Stroke 33, 831-836 (2002).

38. Heo, J. H. et al. Matrix metalloproteinases increase very early during experimental focal cerebral ischemia. J. Cereb. Blood Flow Metab. 19, 624-633 (1999).

39. Asahi, M., Sumii, T., Fini, M. E., Itohara, S. \& Lo, E. H. Matrix metalloproteinase 2 gene knockout has no effect on acute brain injury after focal ischemia. Neuroreport 12, 3003-3007 (2001).

40. Montaner, J. et al. Matrix metalloproteinase- 9 pretreatment level predicts intracranial hemorrhagic complications after thrombolysis in human stroke. Circulation 107, 598-603 (2003).

41. Castellanos, M. et al. Plasma metalloproteinase- 9 concentration predicts hemorrhagic transformation in acute ischaemic stroke. Stroke 34, 40-46 (2003).

42. Harris, A. K. et al. Effect of neutrophil depletion on gelatinase expression, oedema formation and hemorrhagic transformation after focal ischaemic stroke. BMC Neurosci. 6, 49 (2005).

43. Hacke, W. et al. Thrombolysis with alteplase 3 to 4.5 hours after acute ischaemic stroke. N. Engl. J. Med. 359, 1317-1329 (2008).

44. Toni, D. et al. Hemorrhagic transformation of brain infarct: predictability in the first 5 hours from stroke onset and influence on clinical outcome. Neurology 46, 341-345 (1996).

45. Hacke, W. et al. 'Malignant' middle cerebral artery territory infarction: clinical course and prognostic signs. Arch. Neurol. 53, 309-315 (1996).

46. Wagner, J. C. \& Lutsep, H. L. Thrombolysis in young adults. J. Thromb. Thrombolysis 20, 133-136 (2005).

47. Liu, F., Yuan, R., Benashski, S. E. \& McCullough, L. D. Changes in experimental stroke outcome across the life span. J. Cereb. Blood Flow Metab. 29, 792-802 (2009).

48. Manno, E. M., Adams, R. E., Derdeyn, C. P., Powers, W. J. \& Diringer, M. N. The effects of mannitol on cerebral oedema after large hemispheric cerebral infarct. Neurology 52, 583-587 (1999).

49. Schwab, S., Spranger, M., Schwarz, S. \& Hacke, W. Barbiturate coma in severe hemispheric stroke: useful or obsolete? Neurology 48, 1608-1613 (1997).

50. Bardutzky, J. \& Schwab, S. Antiedema therapy in ischaemic stroke. Stroke 38, 3084-3094 (2007).

51. Vahedi, K. et al. Early decompressive surgery in malignant infarction of the middle cerebral artery: a pooled analysis of three randomised controlled trials. Lancet Neurol. 6, 215-222 (2007).

52. Faraci, F. M. Vascular protection. Stroke 34, 327-329 (2003).

53. Fagan, S. C., Hess, D. C., Hohnadel, E. J., Pollock, D. M. \& Ergul, A. Targets for vascular protection after acute ischaemic stroke. Stroke 35, 2220-2225 (2004).

54. Palomares, S. M. \& Cipolla, M. J. Vascular protection following cerebral ischemia and reperfusion. J. Neurol. Neurophysiol. 2011, S1-004 (2011).

55. Cruz-Orengo, L. et al. Enhanced sphingosine-1-phosphate receptor 2 expression underlies female CNS autoimmunity susceptibility. J. Clin. Invest. 124, 2571-2584 (2014).
56. Hla, T. \& Maciag, T. An abundant transcript induced in differentiating human endothelial cells encodes a polypeptide with structural similarities to G-proteincoupled receptors. J. Biol. Chem. 265, 9308-9313 (1990).

57. Mandala, S. et al. Alteration of lymphocyte trafficking by sphingosine-1phosphate receptor agonists. Science 296, 346-349 (2002).

58. Allende, M. L., Dreier, J. L., Mandala, S. \& Proia, R. L. Expression of the sphingosine 1-phosphate receptor, S1P1, on T-cells controls thymic emigration J. Biol. Chem. 279, 15396-15401 (2004).

59. Wei, Y. et al. Fingolimod provides long-term protection in rodent models of cerebral ischemia. Ann. Neurol. 69, 119-129 (2011).

60. Kraft, P. et al. FTY720 ameliorates acute ischaemic stroke in mice by reducing thrombo-inflammation but not by direct neuroprotection. Stroke $\mathbf{4 4}$, 3202-3210 (2013).

61. Kappos, L. et al. A placebo-controlled trial of oral fingolimod in relapsing multiple sclerosis. N. Engl. J. Med. 362, 387-401 (2010).

62. Vogelgesang, A. et al. Analysis of lymphocyte subsets in patients with stroke and their influence on infection after stroke. Stroke 39, 237-241 (2008).

63. Dirnagl, U. et al. Stroke-induced immunodepression: experimental evidence and clinical relevance. Stroke 38, 770-773 (2007).

64. Xia, P. et al. Tumor necrosis factor-alpha induces adhesion molecule expression through the sphingosine kinase pathway. Proc. Natl Acad. Sci. USA 95, 14196-14201 (1998).

65. Olivera, A. et al. Sphingosine kinase 1 and sphingosine-1-phosphate receptor 2 are vital to recovery from anaphylactic shock in mice. J. Clin. Invest. 120, 1429-1440 (2010).

66. Cui, H. et al. Sphingosine-1-phosphate receptor 2 protects against anaphylactic shock through suppression of endothelial nitric oxide synthase in mice. J. Allergy Clin. Immunol. 132, 1205-1214 e1209 (2013).

67. McCullough, L. D. et al. Pharmacological inhibition of AMP-activated protein kinase provides neuroprotection in stroke. J. Biol. Chem. 280, 20493-20502 (2005).

68. Kim, G. S., Jung, J. E., Niizuma, K. \& Chan, P. H. CK2 is a novel negative regulator of NADPH oxidase and a neuroprotectant in mice after cerebral ischemia. J. Neurosci. 29, 14779-14789 (2009).

69. Shih, S. C. \& Smith, L. E. Quantitative multi-gene transcriptional profiling using real-time PCR with a master template. Exp. Mol. Pathol. 79, 14-22 (2005).

70. Lapchak, P. A., Zhang, J. H. \& Noble-Haeusslein, L. J. RIGOR guidelines: escalating STAIR and STEPS for effective translational research. Transl. Stroke Res. 4, 279-285 (2013).

\section{Acknowledgements}

This work was supported by funds provided by the Departments of Surgery and Emergency Medicine (BIDMC), American Heart Association Grant-in-Aid 12GRNT12050110 and NIH grant HL094465 (to T.S.). We would like to thank Dan L and Dr Shou-ching Jaminet from the Multi-Gene Transcriptional Profiling Core Facility (the Center for Vascular Biology Research, Beth Israel Deaconess Medical Center, Harvard Medical School) for the quantitative PCR analysis, Dr Richard Proia (NIDDK) for providing the S1pr2 $2^{-/}$mice and Dr Timothy Hla (Cornell University) for the $\beta$-galactosidase and S1PR2 adenoviruses.

\section{Author contributions}

G.S.K., L.Y. and M.J.K. performed research, collected the data, analysed the data and wrote the manuscript. G.Z. and H.Z. performed research, collected the data and analysed the data. L.D.M. and M.S. edited the manuscript. T.S. designed experiments, performed research, analysed the data, interpreted the data and wrote the manuscript.

\section{Additional information}

Supplementary Information accompanies this paper at http://www.nature.com/ naturecommunications

Competing financial interests: The authors declare no competing financial interests

Reprints and permission information is available online at http://npg.nature.com/ reprintsandpermissions/

How to cite this article: Kim, G. S. et al. Critical Role of sphingosine-1-phosphate receptor-2 in the Disruption of Cerebrovascular Integrity in Experimental Stroke. Nat. Commun. 6:7893 doi: 10.1038/ncomms8893 (2015).

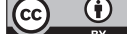

This work is licensed under a Creative Commons Attribution 4.0 International License. The images or other third party material in this article are included in the article's Creative Commons license, unless indicated otherwise in the credit line; if the material is not included under the Creative Commons license, users will need to obtain permission from the license holder to reproduce the material. To view a copy of this license, visit http://creativecommons.org/licenses/by/4.0/ 